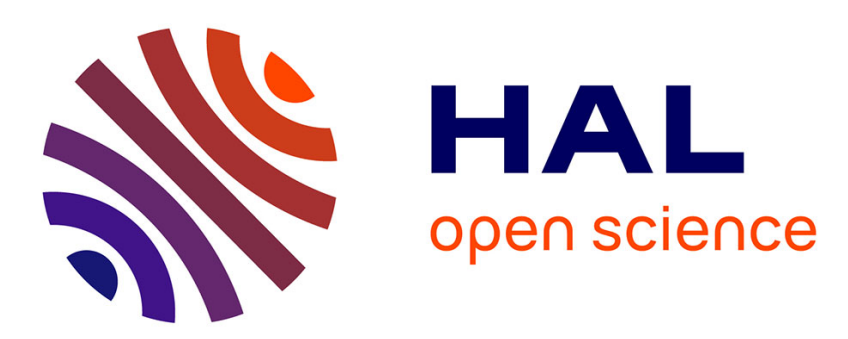

\title{
Second-order multivalued stochastic differential equations on Riemannian manifolds
}

Frédéric Bernardin, Michelle Schatzman, Claude-Henri Lamarque

\section{To cite this version:}

Frédéric Bernardin, Michelle Schatzman, Claude-Henri Lamarque. Second-order multivalued stochastic differential equations on Riemannian manifolds. Proceedings of the Royal Society A: Mathematical, Physical and Engineering Sciences, 2004, 460 (2051), pp.3095-3121. 10.1098/rspa.2004.1312 . hal01435465

\section{HAL Id: hal-01435465 \\ https://hal.science/hal-01435465}

Submitted on 14 Jan 2017

HAL is a multi-disciplinary open access archive for the deposit and dissemination of scientific research documents, whether they are published or not. The documents may come from teaching and research institutions in France or abroad, or from public or private research centers.
L'archive ouverte pluridisciplinaire HAL, est destinée au dépôt et à la diffusion de documents scientifiques de niveau recherche, publiés ou non, émanant des établissements d'enseignement et de recherche français ou étrangers, des laboratoires publics ou privés. 


\title{
Second-order multivalued stochastic differential equations on Riemannian manifolds
}

\author{
By Frédéric Bernardin ${ }^{1}$, Michelle SCHAtzman ${ }^{2}$ And \\ CLAUDE-HENRI LAMARQUE ${ }^{1}$ \\ ${ }^{1}$ Ecole Nationale des Travaux Publics de l'Etat, Laboratoire GéoMatériaux, \\ DGCB URA CNRS 1652, 2 rue Maurice Audin, \\ 69518 Vaulx-en-Velin CEDEX, France (frederic.bernardin@wanadoo.fr) \\ ${ }^{2}$ Université Claude Bernard Lyon I, Laboratoire d'analyse numérique-UMR \\ CNRS 5585, 43 bd. du 11 Novembre 1918, 69622 Villeurbanne CEDEX, France
}

The existence and uniqueness of solutions to multivalued stochastic differential equations of the second order on Riemannian manifolds are proved. The class of problem is motivated by rigid body and multibody dynamics with friction and an application to the spherical pendulum with friction is presented.

Keywords: stochastic differential equations; manifolds; friction; differential inclusions; maximal monotone operators; rigid body dynamics

\section{Introduction}

In the theory of rigid bodies mechanics, a system is described by the configuration manifold $M$, which is generally the set of isometries of the three-dimensional space; the movement of the body is then a curve on this manifold. The kinetic energy defines a Riemannian metric and the equation of motion is a differential equation on a Riemannian manifold. If a stochastic term is involved in the forces acting on the system, stochastic differential equations must be used and we refer to Ikeda \& Watanabe (1989), Norris (1992) and Emery (1989) for information. We are interested in multivalued maximal monotone operators, since our physical model includes friction. The friction force $f$ is of physical origin. Mathematically, it belongs to the cotangent space at $x$ to $M$, since it is a generalized force. Moreover, it must belong to a closed convex subset $C(x)$ of the cotangent space $T_{x}^{*} M$, and it is related to the velocity through the principle of maximal dissipation, stated for instance by Ballard (2002) under the form

$$
\forall g \in C(x), \quad-\langle f, \dot{x}\rangle_{x} \geqslant-\langle g, \dot{x}\rangle_{x}
$$

where $\langle\cdot, \cdot\rangle_{x}$ denotes the scalar product between $T_{x}^{*} M$ and the tangent space at $x$, $T_{x} M$. It is equivalent to writing $f \in-\partial \psi_{C(x)}(\dot{x})$, where $\psi_{C(x)}$ is a convex homogeneous function of degree 1 which will be defined later, and $\partial$ denotes the subdifferential. It is well known that the subdifferential of such a convex function is a multivalued maximal monotone operator. This the reason why we are interested in second-order multivalued stochastic differential equations (MSDEs) on Riemannian 
manifolds. We prove existence and uniqueness under the classical assumptions: Lipschitz continuity and linear growth of the drift and diffusion coefficients (see Mao (1997, p. 51) for classical stochastic differential equations).

Let us observe that it is not easy to give a meaning to a first-order multivalued differential equation on a Riemannian manifold; indeed, assume that in a local chart the equation would be of the type

$$
\frac{\mathrm{d} x}{\mathrm{~d} t}+A(t, x) \ni f(t)
$$

We have to understand $\mathrm{d} x / \mathrm{d} t$ as an element of the tangent space at $x(t), T_{x(t)} M$ and, therefore, $f(t)$ must belong to the same space and $A(t, x)$ must be a subset of this space. If we would like to state a monotonicity assumption on $A(t, \cdot)$, it is not difficult to transport the elements of $A\left(t, x_{1}\right)$ by parallel transport to the tangent space at $x_{2}$; however, monotonicity is a concept which requires duality between vector spaces. The difference $x_{1}-x_{2}$ cannot be assigned an intrinsic meaning and, therefore, the main principle of differential geometry, that is, chart independence, cannot be upheld. This objection fails if, instead of working on a manifold $M$, we work on its tangent space $T M$, which is the space of classical mechanics, our present setting.

In a local chart, the simplest equation of mechanics, that is, the equation of geodesics, contains a quadratic term of geometric origin. In order to apply the results of Cépa (1995), we have to get rid of this quadratic term, and we can do that by a parallel transport of the equation back to the initial position. Unfortunately, we have to pay for this transformation, since now the coefficients of the new equation depend on the history up to time $t$ of the position, i.e. the restriction $x /[0, t]$ of $x$ to $[0, t]$. The initial formulation is Markovian, the transformation by parallel transport does not have to be Markovian, the construction in the tangent space at the initial point is not Markovian, but the reciprocal transformation which enables us to return to the initial formulation takes care of all these difficulties.

The paper is organized as follows. In $\S 2$, we give definitions and assumptions and define the problem. Working in a local chart is equivalent to working in an open subset of $\mathbb{R}^{d}$; in $\S 3$ we extend the coefficients of the equation to all of $\mathbb{R}^{d}$ and we will denote these coefficients $\tilde{b}, \tilde{\nu}, \tilde{\sigma}$ and $\tilde{A}$. Unfortunately, they are only locally Lipschitz continuous with respect to the velocity $v$. We define new coefficients which have better properties; typically, $\sigma_{n}(t, x, v)$ is equal to $\tilde{\sigma}(t, x, v)$ if $\|v\| \leqslant n$, and is Lipschitz continuous if $\|v\| \geqslant n$. There may exist a geometrical proof showing that $\tilde{b}$ is Lipschitz continuous, under appropriate assumptions on the curvature tensor but this proof will not be presented in this article. In $\S 4$, we apply the results of Cépa (1995) to prove the existence and uniqueness of a solution of our equation with coefficients $b_{n}, \sigma_{n}$ and $\nu_{n}$. In $\S 5$, we prove convergence for $n$ tending to infinity by stopping-time techniques. In $\S 6$, we patch together the local solutions. Finally, an illustrative example is given in $\S 7$ : the spherical pendulum.

Throughout this article, the Einstein summation convention is used, the time derivative of a map $f$ is denoted by $\dot{f}$ and the value of $f$ at the point $t$ is denoted indifferently by $f(t)$ or $f_{t}$.

\section{Definitions}

We consider a smooth Riemannian manifold $M$ of dimension $d$ and we denote by $d_{M}$ the Riemannian metric on $M$ and by $B_{M}(x, \varepsilon)$ (respectively $\bar{B}_{M}(x, \varepsilon)$ ) the open 
ball $\left\{y \in M, d_{M}(x, y)<\varepsilon\right\}$ (respectively the closed ball $\left\{y \in M, d_{M}(x, y) \leqslant \varepsilon\right\}$ ). We suppose the following.

Assumption 2.1. There exist $\left.r>0, r^{\prime} \in\right] 0, r[$ and a finite or countable number of points $z_{k}$ in $M$, such that $M$ can be covered by balls $B_{M}\left(z_{k}, r^{\prime}\right)$ of radius $r^{\prime}$ about $z_{k}$ and each ball of radius $r$ about $z_{k}$ is totally included in one chart of $M$. Moreover, this covering can be taken to be locally finite.

In particular, this assumption is verified if $M$ is a compact manifold or if its curvature tensor is bounded. A curve on $M$ is a map $\rho$ of class $C^{1}$ from $[0, T]$ to $M$ $(T>0)$ and we denote by $\tau\left(\rho_{/[a, b]}\right)$ the parallel transport along $\rho_{/[a, b]}$ from $T_{\rho(a)} M$ to $T_{\rho(b)} M$. We recall that $\tau\left(\rho_{/[a, b]}\right)$ is an isometry between $T_{\rho(a)} M$ and $T_{\rho(b)} M$ (see Abraham \& Marsden (1985) for a proof) and is independent of the parametrization of $\rho$. We need also the important particular case of geodesics: assuming that the geodesic from $x_{1} \in M$ to $x_{2} \in M$ is unique $\bar{\tau}\left(x_{1}, x_{2}\right)$ is the parallel transport from $T_{x_{1}} M$ to $T_{x_{2}} M$. We denote by $\pi: T M \rightarrow M$ the projection map of the tangent bundle $T M$ of $M$.

In order to describe the class of maximal monotone operators used here, we observe first that, for each $x \in M$, a maximal monotone graph relative to the tangent scalar product $(\cdot, \cdot)_{x}$ in the fibre $T_{x} M$ can be defined in a straightforward fashion: such a graph is a subset $A_{x}^{\prime}$ of $\left(T_{x} M\right)^{2}$ such that for all pairs $\left(v_{1}, w_{1}\right)$ and $\left(v_{2}, w_{2}\right)$ in $A_{x}^{\prime}$,

$$
\left(v_{1}-v_{2}, w_{1}-w_{2}\right)_{x} \geqslant 0 \text { (monotonicity) }
$$

and $A_{x}^{\prime}$ is maximal for inclusion among all monotone graphs. In consequence and, without being too formal, there is a bundle of maximal monotone graphs on the manifold $M$ : if $T^{2} M$ denotes the bundle whose generic fibre is $T_{x}^{2} M=\left(T_{x} M\right)^{2}$, the fibre of the bundle of maximal monotone graphs is simply the set of maximal monotone graphs in $T_{x}^{2} M$. In order to avoid repetitions we will even assume that this $A_{x}^{\prime}$ also depends on the time $t \in[0, T]$ so that we consider for each $t \in[0, T]$ and $x \in M$ a maximal monotone graph $A^{\prime}(t, x)$ in $T_{x}^{2} M$. Assumption 2.5 describes the regularity of $A^{\prime}(t, x)$ with respect to $t$ and $x$. For all $t, x$ and $v \in T_{x} M$, we denote by $A^{\prime}(t, x, v)$ the image by $A^{\prime}(t, x)$ of $v$, which is a closed convex set. We suppose that the domain of $A^{\prime}(t, x)$ is all $T_{x} M$ for all $0 \leqslant t \leqslant T$ and $x \in M$, i.e. for all $t$ in $[0, T]$ and all $x$ in $M$ and all $v$ in $T_{x} M, A^{\prime}(t, x, v)$ is not empty. Given $w \in T_{x} M$, we denote by $A^{\prime}(t, x, v, w)$ the projection of $w$ onto $A^{\prime}(t, x, v)$ relative to the metric of $T_{x} M$.

We denote $\mathbb{R}^{d}$ by $E$. If $F$ and $G$ are two finite-dimensional vector spaces, we denote by $L(F, G)$ the set of linear maps from $F$ into $G$. For $t \in[0, T]$ and $x \in M$, let the drift $\nu^{\prime}(t, x)$ be a map from $T_{x} M$ into $T_{x} M$ and let the diffusion matrix $\sigma^{\prime}(t, x)$ be a differentiable map from $T_{x} M$ into $L\left(E, T_{x} M\right)$. For all $v \in T_{x} M$, let us define

$$
R(t, x, v)=\left\|\sigma^{\prime}(t, x, v)\right\|_{L\left(E, T_{x} M\right)}^{2} .
$$

Here $\sigma^{\prime}(t, x, v)$ and $\nu^{\prime}(t, x, v)$ are, respectively, the image of $v$ by $\sigma^{\prime}(t, x)$ and by $\nu^{\prime}(t, x)$. Our assumption on the drift and the diffusion are tailored for a Stratonovich stochastic differential system; for this reason the square of the diffusion matrix appears in assumptions 2.2 and 2.3.

We denote by $\ell$ a continuous map from $[0 ; T]$ to $\mathbb{R}_{+}$such that $\lim _{t \rightarrow 0} \ell(t)=0$. We suppose the following assumptions. 
Assumption 2.2. For all bounded open subset $U \subset M$, there exists $C_{0}(U)$ such that for all close enough $x$ and $y$ in $U$, for all $v$ in $T_{x} M$ and $u$ in $T_{y} M$, and for all $s$ and $t$ in $[0, T]$,

$$
\left|\frac{\partial R}{\partial v}(t, x, v)-\frac{\partial R}{\partial v}(s, y, u)\right| \leqslant C_{0}(U)\left(\ell(|t-s|)+d_{M}(x, y)+\|v-\bar{\tau}(y, x) u\|_{x}\right) .
$$

Assumption 2.3. There exists a constant $C_{1}$ such that for all $0 \leqslant t \leqslant T$ and for all $(x, v) \in T M$,

$$
\left\|\nu^{\prime}(t, x, v)\right\|_{x}+\left\|\sigma^{\prime}(t, x, v)\right\|_{L\left(E, T_{x} M\right)}+\left|\frac{\partial R}{\partial v}(t, x, v)\right| \leqslant C_{1}\left(1+d_{M}\left(x, x_{0}\right)+\|v\|_{x}\right) .
$$

Here $x_{0}$ is fixed in $M$.

Assumption 2.4. For all bounded open subset $U \subset M$, there exists $C_{2}(U)$ such that for all close enough $x$ and $y$ in $U$, for all $v$ in $T_{x} M$ and $u$ in $T_{y} M$, and for all $s$ and $t$ in $[0, T]$,

$$
\begin{aligned}
\left\|\nu^{\prime}(t, x, v)-\bar{\tau}(y, x) \nu^{\prime}(s, y, u)\right\|_{x}+ & \left\|\sigma^{\prime}(t, x, v)-\bar{\tau}(y, x) \circ \sigma^{\prime}(s, y, u)\right\|_{L\left(E, T_{x} M\right)} \\
& \leqslant C_{2}(U)\left(\ell(|t-s|)+d_{M}(x, y)+\|v-\bar{\tau}(y, x) u\|_{x}\right) .
\end{aligned}
$$

Assumption 2.5. For all bounded open subset $U \subset M$, there exists $C_{3}(U)$ such that for all close enough $x$ and $y$ in $U$, for all $v$ and $w_{1}$ in $T_{x} M$ and $u$ and $w_{2}$ in $T_{y} M$, and for all $s$ and $t$ in $[0, T]$,

$$
\begin{aligned}
& \left\|A^{\prime}\left(t, x, v, w_{1}\right)-\bar{\tau}(y, x) A^{\prime}\left(s, y, u, w_{2}\right)\right\|_{x} \\
& \quad \leqslant C_{3}(U)\left(\ell(|t-s|)+d_{M}(x, y)+\|v-\bar{\tau}(y, x) u\|_{x}+\left\|w_{1}-\bar{\tau}(y, x) w_{2}\right\|_{x}\right) .
\end{aligned}
$$

Remark 2.6. Assumption 2.5 has an immediate consequence: $A^{\prime}(t, x, v)$ is locally bounded with respect to $v$, uniformly with respect to $t \in[0, T]$ and to $x$ in compact subsets of $M$.

We consider $\mathcal{E}^{\prime}=\left(\Omega, \mathcal{F},\left(\mathcal{F}_{t}^{\prime}\right)_{t \geqslant 0}, \mathbb{P}\right)$ a filtered probability space which verifies the usual conditions (see Karatzas \& Shreve (1991) for a definition). Let $\left(B_{t}\right)_{t \geqslant 0}$ be the standard $\mathbb{R}^{d}$-Brownian motion on $\mathcal{E}^{\prime}$. Let $\delta$ be the symbol for the Stratonovich differentiation. Let $T_{0}$ be a $\left(\mathcal{F}_{t}^{\prime}\right)$-stopping time such that $T_{0} \leqslant T$ almost surely. Let $\eta^{\prime}$ be a random variable $\mathcal{F}_{T_{0}}^{\prime}$-measurable which takes its values in $T M$, whose secondorder moment, written in a local chart of $M$, is finite. If $\left(v_{t}\right)_{t \geqslant 0}$ is a $T M$-valued continuous semi-martingale over the curve $\rho:[0, T] \rightarrow M$ (a stochastic process $X$ on a manifold $Q$ is a continuous semi-martingale on $Q$ if $f \circ X$ is a real continuous semi-martingale for every $C^{\infty}$ map $f: Q \rightarrow \mathbb{R}$ : see Emery (1989) for details), we denote by $D_{\dot{\rho}(t)} v_{t}$ the Stratonovich covariant differential of $v$ along $\rho$ at time $t$ (see Norris (1992) for a definition). We consider also the MSDE on the manifold $M$ :

$$
\left.\begin{array}{l}
\delta x_{t}^{\prime}=v_{t}^{\prime} \mathrm{d} t, \\
D_{\dot{x}^{\prime}(t)} v_{t}^{\prime}+A^{\prime}\left(t, x_{t}^{\prime}, v_{t}^{\prime}\right) \mathrm{d} t \ni \nu^{\prime}\left(t, x_{t}^{\prime}, v_{t}^{\prime}\right) \mathrm{d} t+\sigma^{\prime}\left(t, x_{t}^{\prime}, v_{t}^{\prime}\right) \delta B_{t}, \quad T_{0} \leqslant t<T, \\
\left(x^{\prime}\left(T_{0}\right), v^{\prime}\left(T_{0}\right)\right)=\left(\pi\left(\eta^{\prime}\right), \eta^{\prime}\right) \text { a.s. }
\end{array}\right\}
$$

The first equation of (2.1) shows that $x_{t}^{\prime}$ is almost surely of class $C^{1}$ on $\left[T_{0}, T[\right.$. For $t \in\left[T_{0}, T\right]$, we apply parallel transport along the trajectories' $x^{\prime}$ solution of $(2.1)$, and 
Norris (1992) has proved that the covariant differential is mapped to Stratonovich differential yielding then the following system:

$$
\begin{aligned}
& \tau\left(x_{/\left[T_{0}, t\right]}^{\prime}\right)^{-1} \delta x_{t}^{\prime}=\left(\tau\left(x_{/\left[T_{0}, t\right]}^{\prime}\right)\right)^{-1} v_{t}^{\prime} \mathrm{d} t, \\
& \delta\left(\left(\tau\left(x_{/\left[T_{0}, t\right]}^{\prime}\right)\right)^{-1} v_{t}^{\prime}\right)+\left(\tau\left(x_{/\left[T_{0}, t\right]}^{\prime}\right)\right)^{-1} A^{\prime}\left(t, x_{t}^{\prime}, v_{t}^{\prime}\right) \mathrm{d} t \\
& \quad \ni\left(\tau\left(x_{/\left[T_{0}, t\right]}^{\prime}\right)\right)^{-1} \nu^{\prime}\left(t, x_{t}^{\prime}, v_{t}^{\prime}\right) \mathrm{d} t\left\{\left(\tau\left(x_{/\left[T_{0}, t\right]}^{\prime}\right)\right)^{-1} \circ \sigma^{\prime}\left(t, x_{t}^{\prime}, v_{t}^{\prime}\right)\right\} \delta B_{t}, \\
& \left(x^{\prime}\left(T_{0}\right), v^{\prime}\left(T_{0}\right)\right)=\left(\pi\left(\eta^{\prime}\right), \eta^{\prime}\right) \text { a.s. }
\end{aligned}
$$

Let $V$ (respectively, $V^{\prime}$ ) a bounded open subset of $E$ (respectively, of $M$ ) such that $\varphi: V^{\prime} \rightarrow V$ define a local chart of $M$ and put $\psi=\varphi^{-1}$. Let $x_{0}$ be a point of $V^{\prime}$ and we identify $T_{x_{0}} M$ and $E=\mathbb{R}^{d}$. Without loss of generality, we suppose that $\varphi\left(x_{0}\right)=0$ and $T_{x_{0}} \varphi=I_{E}$, where $T_{y} \varphi$ denotes the tangent mapping to $\varphi$ at the point $y \in M$ and $I_{E}$ denotes the identity map of $E$. Let $W^{\prime} \subset V^{\prime}$ be an open set of $M$ such that each pair of points of $W^{\prime}$ can be linked by a unique geodesic of $M$. We define $W=\varphi\left(W^{\prime}\right)$. We will define now all the transported objects; for all the primed notation, the corresponding non-primed notation denote a transported object. For $0 \leqslant s \leqslant t \leqslant T$ we denote by $C_{W}^{1}([s, t], V)$ the set of the maps $\xi:[s, t] \rightarrow V$ of class $C^{1}$ such that $\xi(0) \in W$. For $\xi$ an element of $C_{W}^{1}([s, t], V), \psi(\xi)$ is a curve on $V^{\prime}$, and for $v \in V$, we define

$$
b(s, t, \xi, v)=T_{\psi\left(\xi_{t}\right)} \varphi \tau(\psi(\xi)) \bar{\tau}\left(x_{0}, \psi(\xi)(s)\right) v .
$$

We define

$$
\begin{aligned}
& A(s, t, \xi, v)=\bar{\tau}\left(\psi(\xi)(s), x_{0}\right)(\tau(\psi(\xi)))^{-1} A^{\prime}\left(t, \psi\left(\xi_{t}\right), \tau(\psi(\xi)) \bar{\tau}\left(x_{0}, \psi(\xi)(s)\right) v\right), \\
& \nu(s, t, \xi, v)=\bar{\tau}\left(\psi(\xi)(s), x_{0}\right)(\tau(\psi(\xi)))^{-1} \nu^{\prime}\left(t, \psi\left(\xi_{t}\right), \tau(\psi(\xi)) \bar{\tau}\left(x_{0}, \psi(\xi)(s)\right) v\right),
\end{aligned}
$$

and for all $z \in E$,

$$
\sigma(s, t, \xi, v) z=\bar{\tau}\left(\psi(\xi)(s), x_{0}\right)(\tau(\psi(\xi)))^{-1}\left[\sigma^{\prime}\left(t, \psi\left(\xi_{t}\right), \tau(\psi(\xi)) \bar{\tau}\left(x_{0}, \psi(\xi)(s)\right) v\right)\right] z .
$$

Remark 2.7. It is convenient to express the transport in $A, \nu$ and $\sigma$ only through $b$. Let us explain how to do this on $\nu$ : if $S=T_{\psi(\xi(t))} \varphi$, we introduce $S S^{-1}$ at two places in the definition of $\nu(s, t, \xi, v)$ :

$$
\begin{aligned}
& \nu(s, t, \xi, v) \\
& \quad=\bar{\tau}\left(\psi(\xi)(s), x_{0}\right)(\tau(\psi(\xi)))^{-1} S^{-1} S \nu^{\prime}\left(t, \psi\left(\xi_{t}\right), S^{-1} S \tau(\psi(\xi)) \bar{\tau}\left(x_{0}, \psi(\xi)(s)\right) v\right) .
\end{aligned}
$$

Since $S \tau(\psi(\xi)) \bar{\tau}\left(x_{0}, \psi(\xi)(s)\right)=b(s, t, \xi),(2.7)$ is equivalent to

$$
\nu(s, t, \xi, v)=(b(s, t, \xi))^{-1} S \nu^{\prime}\left(t, \psi\left(\xi_{t}\right), S^{-1} b(s, t, \xi, v)\right) .
$$

By a legitimate abuse of notation corresponding to the choice of a local chart, we may drop $\psi\left(\xi_{t}\right)$ and $S=T_{\psi(\xi(t))} \varphi$ in (2.8), yielding thus

$$
\nu(s, t, \xi, v)=b(s, t, \xi)^{-1} \nu^{\prime}\left(t, \xi_{t}, b(s, t, \xi, v)\right) .
$$

There are analogous formulae for $\sigma$ and $A$ :

$$
\sigma(s, t, \xi, v)=b(s, t, \xi)^{-1} \circ \sigma^{\prime}\left(t, \xi_{t}, b(s, t, \xi, v)\right) .
$$


and

$$
A(s, t, \xi, v)=b(s, t, \xi)^{-1} A^{\prime}\left(t, \xi_{t}, b(s, t, \xi, v)\right) .
$$

We suppose in a first time that $\pi(\eta)$ belongs to $W$ almost surely. By defining for all $T_{0} \leqslant t \leqslant T$,

$$
\begin{aligned}
\bar{x}(t) & =\varphi\left(x_{t}^{\prime}\right), \\
\bar{v}(t) & =\bar{\tau}\left(x^{\prime}\left(T_{0}\right), x_{0}\right)\left(\tau\left(x_{/\left[T_{0}, t\right]}^{\prime}\right)\right)^{-1} v_{t}^{\prime}, \\
\bar{\eta} & =\bar{\tau}\left(x^{\prime}\left(T_{0}\right), x_{0}\right) \eta
\end{aligned}
$$

we have

$$
\begin{aligned}
& \mathrm{d} \bar{x}_{t}=b\left(T_{0}, t, \bar{x}_{/\left[T_{0}, t\right]}, \bar{v}_{t}\right) \mathrm{d} t \\
& \delta \bar{v}_{t}+A\left(T_{0}, t, \bar{x}_{/\left[T_{0}, t\right]}, \bar{v}_{t}\right) \mathrm{d} t \\
& \quad \quad \ni\left(T_{0}, t, \bar{x}_{/\left[T_{0}, t\right]}, \bar{v}_{t}\right) \mathrm{d} t+\sigma\left(T_{0}, t, \bar{x}_{/\left[T_{0}, t\right]}, \bar{v}_{t}\right) \delta B_{t}, T_{0} \leqslant t, \\
& \left(\bar{x}\left(T_{0}\right), \bar{v}\left(T_{0}\right)\right)=(\pi(\bar{\eta}), \bar{\eta}) \text { a.s. }
\end{aligned}
$$

Finally, we define $\left(\eta_{1}, \eta_{2}\right)=(\pi(\bar{\eta}), \bar{\eta})$ and $x, v:\left[0, T-T_{0}\right] \rightarrow E$ by

$$
x(t)=\bar{x}\left(t+T_{0}\right) \quad \text { and } \quad v(t)=\bar{v}\left(t+T_{0}\right)
$$

and then, by denoting $b(0, t, \xi, v)$ by $b(t, \xi, v), \nu(0, t, \xi, v)$ by $\nu(t, \xi, v), \sigma(0, t, \xi, v)$ by $\sigma(t, \xi, v)$ and $A(0, t, \xi, v)$ by $A(t, \xi, v)$, we seek the restriction to $\left[0, T-T_{0}\right.$ [ of the solution to the following system:

$$
\begin{aligned}
& \mathrm{d} x_{t}=b\left(t, x_{/[0, t]}, v_{t}\right) \mathrm{d} t, \\
& \delta v_{t}+A\left(t, x_{/[0, t]}, v_{t}\right) \mathrm{d} t \\
& \quad \ni \nu\left(t, x_{/[0, t]}, v_{t}\right) \mathrm{d} t+\sigma\left(t, x_{/[0, t]}, v_{t}\right) \delta W_{t}, \quad 0 \leqslant t \leqslant T, \\
& (x(0), v(0))=\left(\eta_{1}, \eta_{2}\right) \text { a.s. }
\end{aligned}
$$

where $\left(W_{t}\right)_{t \geqslant 0}$ is defined by $W_{t}=B_{t+T_{0}}-B_{T_{0}}$.

For all $t \geqslant 0$, let $\mathcal{F}_{t}=\mathcal{F}_{T_{0}+t}^{\prime}$. It is well known that $\left(W_{t}\right)_{t \geqslant 0}$ is a standard Brownian motion on the filtered probability space $\mathcal{E}=\left(\Omega, \mathcal{F},\left(\mathcal{F}_{t}\right)_{t \geqslant 0}, \mathbb{P}\right)$. We say that $\left(x_{t}, v_{t}\right)_{0 \leqslant t \leqslant T}$ is a solution to $(2.13)$ if $\left(x_{t}, v_{t}\right)_{0 \leqslant t \leqslant T}$ is $\mathcal{F}_{t}$-adapted, $\mathrm{d} x_{t}=$ $b\left(t, x /[0, t], v_{t}\right) \mathrm{d} t$ almost surely and if there exists a stochastic process $\mathcal{F}_{t}$-adapted $\left(K_{t}\right)_{0 \leqslant t \leqslant T}$ taking its values in $E$ with bounded variation on $[0, T]$ such that $K_{0}=0$ almost surely and

$$
v_{t}=v_{0}+\int_{0}^{t} \nu\left(s, x_{/[0, s]}, v_{s}\right) \mathrm{d} s+\int_{0}^{t} \sigma\left(s, x_{/[0, s]}, v_{s}\right) \delta W_{s}-K_{t}
$$

and $\mathrm{d} K_{t} \in A\left(t, x_{/[0, t]}, v_{t}\right) \mathrm{d} t$. The last inclusion has the following sense: the measure $\left(v_{t}-\alpha_{t}, \mathrm{~d} K_{t}-\beta_{t} \mathrm{~d} t\right)_{x_{0}}$ is almost surely non-negative on $[0, T]$ for all choices of continuous maps $\alpha, \beta:[0, T] \rightarrow E$ such that $\beta_{t} \in A\left(t, x_{/[0, t]}, \alpha_{t}\right)$ (see Cépa (1995) for precisions).

Remark 2.8. If $\sigma^{\prime}(t, x, v)$ does not depend on $v$, it is possible to consider the equation (2.1) in the Itô sense - the formulation is intrinsic - and the assumption 2.2 and the linear growth condition 2.3 for $R(t, x, v)$ are then not necessary.

In the following we will denote by $(\cdot, \cdot)$ the scalar product $(\cdot, \cdot)_{x_{0}}$. 


\section{Extension of the problem to $E$}

We are slightly abusive when we say that we extend $b, A, \nu$ and $\sigma$ since we have first to restrict the values of $x$ to a set $U \ni x_{0}$, with $\bar{U}$ a subset of $V$. More precisely, given a smooth cut-off function $\chi$ such that

$$
\chi(y)= \begin{cases}1 & \text { if } y \in U, \\ 0 & \text { if } y \notin V,\end{cases}
$$

the function $s \mapsto \chi\left(x_{s}\right) x_{s}=\tilde{x}_{s}$ coincides with $x_{s}$ as long as $x_{s}$ does not leave $U$. If we use $\tilde{x}_{s}$ instead of $x_{s}$ in the definition of $b, A, \nu$ and $\sigma$, we will prove that the corresponding $\tilde{b}, \tilde{\nu}$ and $\tilde{\sigma}$ are not Lipschitz continuous with respect to $x$ or $v$. Therefore, we will need an extra step, where we project $v$ onto the ball of radius $n$ about 0 . Let us go into the technicalities.

The functions $b, \nu, \sigma$ and $A$ have been respectively defined in (2.3), (2.5), (2.6) and (2.4), which depend on the local chart $\varphi$; therefore all the upcoming constants will also depend on $\varphi$.

Let $U$ be a fixed bounded neighbourhood of $x_{0} \in V$ and assume $\bar{W} \subset U \subset \bar{U} \subset V$. For $B$ an open subset of $E$ containing $x_{0}, \Delta(B)$ is the set of pairs $(t, x)$ with $t \in[0, T]$ and $x$ a function of class $C^{1}$ from $[0, t]$ to $B$, such that $x(0) \in W$ :

$$
\Delta(B)=\left\{(t, x), t \in[0, T], x \in C_{W}^{1}([0, t], B)\right\} .
$$

We denote by $G=\left(g_{i j}\right)_{1 \leqslant i, j \leqslant d}$ the metric tensor whose inverse is denoted by $G^{-1}=\left(g^{i j}\right)_{1 \leqslant i, j \leqslant d}$ and the functions $g_{i j}$ and $g^{i j}$ are smooth on $V$. Let $\left(x^{1}, \ldots, x^{d}\right)$ be the local coordinates of the chart $\varphi$ and $e_{0}=\left(\partial / \partial x^{1}, \ldots, \partial / \partial x^{d}\right)$ be a basis of $E$. For $(t, x) \in \Delta(V)$ and $1 \leqslant i \leqslant d, b\left(t, x, e_{0, i}\right)=e_{i}^{j}(t) e_{0, j}$, where (see Abraham \& Marsden 1985)

$$
e_{i}^{j}(t)=e_{i, 0}^{j}-\int_{0}^{t} \Gamma_{k l}^{j}\left(x_{s}\right) e_{i}^{k}(s) \dot{x}_{s}^{l} \mathrm{~d} s .
$$

The functions

$$
\Gamma_{l k}^{i}(x)=\Gamma_{k l}^{i}(x)=\frac{1}{2} g^{i h}(x)\left(\frac{\partial g_{h k}}{\partial x^{l}}(x)+\frac{\partial g_{h l}}{\partial x^{k}}(x)-\frac{\partial g_{k l}}{\partial x^{h}}(x)\right)
$$

are the Christoffel symbols of $(M, G)$. If we denote by $I_{E}$ the identity matrix of $E$, we can write $b(t, x)^{\mathrm{T}} G\left(x_{t}\right) b(t, x)=I_{E}$ (see Abraham \& Marsden 1985) and then $\operatorname{tr}\left\{b(t, x) b(t, x)^{\mathrm{T}}\right\}=\operatorname{tr}\left\{G^{-1}\left(x_{t}\right)\right\}$, which shows the boundedness of $(t, x) \mapsto$ $b(t, x)$, which can also be seen on the definition of $b$ in (2.3). Let $\chi$ be as in (3.1). Throughout this paper we denote by $\|\cdot\|_{\infty, t}$ the uniform norm on the space $C^{0}([0, t], E)$ of continuous maps from $[0, t]$ to $E, t>0$.

Lemma 3.1. Define $\zeta$ on $C^{0}([0, t], E)$ by $\zeta(u)=\chi(u) u$. Then $\zeta$ is Lipschitz continuous from $C^{0}([0, t], E)$ to itself.

Proof. Clear.

Let $(t, x) \in \Delta(V)$; we first define $t_{x}^{U}$, which is the first exit time out of $U$ :

$$
t_{x}^{U}=\inf \{s \leqslant t, x(s) \notin U\} \wedge T .
$$


We now let

$$
\tilde{x}=\chi(x) x
$$

and $\tilde{x}$ coincides with $x$ on the interval $\left[0, t_{x}^{U}\right]$. Moreover, the following estimates are straightforward:

$$
\|\tilde{x}\|_{\infty, t} \leqslant C_{\varphi}, \quad\|\dot{\tilde{x}}\|_{\infty, t} \leqslant C_{\varphi}\|\dot{x}\|_{\infty, t}
$$

and, if $(t, y) \in \Delta(V)$,

$$
\|\tilde{x}-\tilde{y}\|_{\infty, t}+\|\dot{\tilde{x}}-\dot{\tilde{y}}\|_{\infty, t} \leqslant C_{\varphi}\left(\|\dot{x}\|_{\infty, t}+\|\dot{y}\|_{\infty, t}\right)\left(\|x-y\|_{\infty, t}+\|\dot{x}-\dot{y}\|_{\infty, t}\right),
$$

with $C_{\varphi}$ depending only on $U, V$ and $\varphi$. The verification of these estimates is straightforward and left to the reader.

Definition 3.2. We let, for all $(t, x) \in D(E)$,

$$
\begin{gathered}
\tilde{b}(t, x)=b(t, \tilde{x}), \\
\tilde{\nu}(t, x)=\nu(t, \tilde{x}), \\
\tilde{\sigma}(t, x)=\sigma(t, \tilde{x}), \\
\tilde{A}(t, x)=A(t, \tilde{x}) .
\end{gathered}
$$

Lemma 3.3. The maps $\tilde{b}, \tilde{\nu}$ and $\tilde{\sigma}$ grow at most linearly, that is, for all $((t, x), v) \in$ $\Delta(E) \times E$,

$$
\|\tilde{\nu}(t, x, v)\|+\|\tilde{\sigma}(t, x, v)\|+\|\tilde{b}(t, x, v)\| \leqslant C_{\varphi}\left(\|x\|_{\infty, t}+\|\dot{x}\|_{\infty, t}+\|v\|\right) .
$$

Proof. We know that a constant $C_{\varphi}$ exists so that for all $((t, x), v) \in \Delta(E) \times E$, $\|\tilde{b}(t, x, v)\| \leqslant C_{\varphi}\|v\|$ and $\left\|(\tilde{b}(t, x))^{-1} v\right\| \leqslant C_{\varphi}\|v\|$. Since $\tau(\psi(\tilde{x})) \bar{\tau}\left(x_{0}, \psi(\tilde{x})(0)\right)$ is an isometry between $E$ and $T_{\psi(\tilde{x})(t)} M$ and using assumption 2.3, the result holds.

The functions $\tilde{b}, \tilde{\nu}$ and $\tilde{\sigma}$ are not Lipschitz continuous with respect to $x$ and $v$. We replace them by Lipschitz continuous approximations as follows. For $n \in \mathbb{N}^{*}$, let $\mathcal{U}_{n}=\mathcal{B}(0, n), \mathcal{V}_{n}=\mathcal{B}(0, n+1)$ and $\chi_{n}$ be the map associated to these bounded open sets. All constants depending only on $n$ are denoted by $C_{n}$ and by $C_{\varphi n}$ if they depend on $\varphi$ and $n$. For $(t, x) \in \Delta(E)$, let us define

$$
x_{n}(s)=x(0)+\int_{0}^{s} \chi_{n}(\dot{x}(u)) \dot{x}(u) \mathrm{d} u,
$$

and clearly

$$
\left\|\dot{x}_{n}\right\|_{\infty, t} \leqslant n+1
$$

Using lemma 3.1, for all $(t, y) \in \Delta(E)$, we have

$$
\left\|x_{n}-y_{n}\right\|_{\infty, t}+\left\|\dot{x}_{n}-\dot{y}_{n}\right\|_{\infty, t} \leqslant C_{\varphi n}\left(\|x(0)-y(0)\|+\|\dot{x}-\dot{y}\|_{\infty, t}\right) .
$$

If $t_{x}^{n}=\inf \{s \leqslant t,\|\dot{x}(s)\| \geqslant n\} \wedge T, x_{n}=x$ on $\left[0, t_{x}^{n}\right]$.

Definition 3.4. We let for all $(t, x, v) \in \Delta(E) \times E$,

$$
\begin{aligned}
b_{n}(t, x, v) & =\tilde{b}\left(t, x_{n}, \operatorname{proj}_{\overline{\mathcal{B}}(0, n)} v\right), \\
\nu_{n}(t, x, v) & =\tilde{\nu}\left(t, x_{n}, \operatorname{proj}_{\overline{\mathcal{B}}(0, n)} v\right), \\
\sigma_{n}(t, x, v) & =\tilde{\sigma}\left(t, x_{n}, \operatorname{proj}_{\overline{\mathcal{B}}(0, n)} v\right), \\
A_{n}(t, x, v) & =\tilde{A}\left(t, x_{n}, v\right) .
\end{aligned}
$$


We give now several results on the above introduced functions.

Lemma 3.5. The functions $b_{n}$ and $\tilde{b}$ verify

$$
\forall(t, x) \in \Delta(E), \forall v \in \overline{\mathcal{B}}(0, n), \forall s \leqslant t_{x}^{n}, \quad b_{n}(s, x /[0, s], v)=\tilde{b}\left(s, x_{/[0, s]}, v\right) .
$$

Proof. Indeed by definition of $b_{n}$,

$$
b_{n}\left(s, x_{/[0, s]}, v\right)=\tilde{b}\left(s, x_{n /[0, s]}, \operatorname{proj}_{\overline{\mathcal{B}}(0, n)} v\right)=\tilde{b}\left(s, x_{/[0, s]}, v\right),
$$

since $s \leqslant t_{x}^{n}=\inf \{u \leqslant t,\|\dot{x}(u)\| \geqslant n\}$.

Remark 3.6. With the help of remark 2.7 and the definitions of $\nu_{n}, \sigma_{n}$ and $A_{n}$, the same equality occurs for $\nu_{n}, \sigma_{n}$ and $A_{n}$.

Lemma 3.7. Operators $A(t, x)$ and $A_{n}(t, x)$ are multivalued, maximal and monotone on $E$ for all $(t, x) \in \Delta(E)$.

Proof. This is a direct consequence of the fact that the transport operator is an isometry.

Remark 3.8. The functions $b_{n}, \nu_{n}$ and $\sigma_{n}$ grow at most linearly, thanks to lemma 3.3.

Lemma 3.9. The maps $b_{n}(t, .,),.\left(b\left(t, \widetilde{\cdot}_{n}\right)^{-1}, \nu_{n}(t, .,\right.$.$) and \sigma_{n}(t, . .$.$) are Lipschitz$ continuous functions: for all $((t, x),(t, y), v, u) \in(\Delta(E))^{2} \times E^{2}$,

$$
\begin{aligned}
\left\|b_{n}(t, x, v)-b_{n}(t, y, u)\right\|+ & \left\|\left(b\left(t, \widetilde{x_{n}}\right)\right)^{-1} v-\left(b\left(t, \widetilde{y_{n}}\right)\right)^{-1} u\right\| \\
+\| \nu_{n}(t, x, v)- & \nu_{n}(t, y, u)\|+\| \sigma_{n}(t, x, v)-\sigma_{n}(t, y, u) \| \\
& \quad \leqslant C_{\varphi n}\left(\|x-y\|_{\infty, t}+\|\dot{x}-\dot{y}\|_{\infty, t}+\|v-u\|\right) .
\end{aligned}
$$

Proof. If we write $\tilde{b}(t, x)=E(t)$ and $\tilde{b}(t, y)=F(t)$, by definition of $\tilde{b}$ we have $\dot{E}(t)=L(\tilde{x}, t) E(t)$ and $\dot{F}(t)=L(\tilde{y}, t) F(t)$, where $L(\rho, t)$ maps linearly the set of invertible square matrices of dimension $d \times d$ into itself and is defined by

$$
(L(\rho, s) A)_{i}^{j}=-\Gamma_{k l}^{j}(\rho(s)) A_{i}^{k} \dot{\rho}_{s}^{l}, \quad s \leqslant t,
$$

for all $\rho$ in $\Delta(V)$ and matrix $A$. We begin by writing

$$
\left.\begin{array}{rl}
\left|[L(\tilde{x}, s) E(s)-L(\tilde{y}, s) E(s)]_{i}^{j}\right| & =\left|\Gamma_{k l}^{j}(\tilde{y}(s)) E_{i}^{k}(s) \dot{\tilde{y}}_{s}^{l}-\Gamma_{k l}^{j}(\tilde{x}(s)) E_{i}^{k}(s) \dot{\tilde{x}}_{s}^{l}\right| \\
& \leqslant\left|\left(\Gamma_{k l}^{j}(\tilde{y}(s))-\Gamma_{k l}^{j}(\tilde{x}(s))\right) E_{i}^{k}(s) \dot{\tilde{y}}_{s}\right| \\
& +\left\|\Gamma_{k l}^{j}(\tilde{x}(s)) E_{i}^{k}(s)\left(\dot{\tilde{y}}_{s}-\dot{\tilde{x}}_{s}\right)\right\| \\
& \leqslant C_{\varphi}\left(\|\dot{\tilde{y}}\|_{\infty, t} \cdot\|\tilde{x}-\tilde{y}\|_{\infty, t}+\|\dot{\tilde{y}}-\dot{\tilde{x}}\|_{\infty, t}\right) .
\end{array}\right\}
$$

We comment here on the last line of (3.15): it is the term $\|\dot{\tilde{y}}\|_{\infty, t}\|\tilde{x}-\tilde{y}\|_{\infty, t}$ which prevents $\tilde{b}$ from being Lipschitz continuous with respect to $x$, since $\|\dot{\tilde{y}}\|_{\infty, t}$ does not have to be bounded. There may exist a geometrical proof showing that $\tilde{b}$ is Lipschitz continuous, under appropriate assumptions on the curvature tensor. Let us define $E_{n}(t)=b\left(t, \widetilde{x_{n}}\right)$ and $F_{n}(t)=b\left(t, \widetilde{y_{n}}\right)$. Since

$$
E_{n}(t)=I_{E}-\int_{0}^{t} L\left(\widetilde{x_{n}}, s\right) E_{n}(s) \mathrm{d} s
$$


and

$$
F_{n}(t)=I_{E}-\int_{0}^{t} L\left(\widetilde{y_{n}}, s\right) F_{n}(s) \mathrm{d} s
$$

we have

$$
\begin{aligned}
\left\|E_{n}(s)-F_{n}(s)\right\| \leqslant \int_{0}^{s}\left\|L\left(\widetilde{y_{n}}, u\right) F_{n}(u)-L\left(\widetilde{y_{n}}, u\right) E_{n}(u)\right\| \mathrm{d} u \\
+\int_{0}^{s}\left\|\left(L\left(\widetilde{y_{n}}, u\right)-L\left(\widetilde{x_{n}}, u\right)\right) E_{n}(u)\right\| \mathrm{d} u,
\end{aligned}
$$

and then

$$
\begin{aligned}
\sup _{0 \leqslant s \leqslant t}\left\|E_{n}(s)-F_{n}(s)\right\| \leqslant \sup _{0 \leqslant s \leqslant t} & \left\|L\left(\widetilde{y_{n}}, s\right)\right\| \int_{0}^{t} \sup _{0 \leqslant u \leqslant s}\left\|E_{n}(u)-F_{n}(u)\right\| \mathrm{d} s \\
& +C_{\varphi}\left(\left\|\dot{\overrightarrow{y_{n}}}\right\|_{\infty, t}\left\|\widetilde{x_{n}}-\widetilde{y_{n}}\right\|_{\infty, t}+\left\|\dot{\overline{y_{n}}}-\dot{\overrightarrow{x_{n}}}\right\|_{\infty, t}\right) .
\end{aligned}
$$

Using (3.2), (3.8) and the continuity of the functions $\left(\Gamma_{k l}^{j}\right)_{k, l, j}$, we have

$$
\sup _{0 \leqslant s \leqslant t}\left\|L\left(\widetilde{y_{n}}, s\right)\right\| \leqslant C_{\varphi n}\left\|\dot{\widetilde{y_{n}}}\right\|_{\infty, t} \leqslant C_{\varphi n}(n+1) .
$$

Using the Gronwall's lemma and inequalities (3.2), (3.3), (3.8), (3.9), we have

$$
\left\|E_{n}(t)-F_{n}(t)\right\| \leqslant C_{\varphi n}\left(\|x-y\|_{\infty, t}+\|\dot{y}-\dot{x}\|_{\infty, t}\right) .
$$

This last inequality and the definition of $b_{n}$ lead to:

$$
\left\|b_{n}(t, x, v)-b_{n}(t, y, u)\right\| \leqslant C_{\varphi n}\left(\|x-y\|_{\infty, t}+\|\dot{x}-\dot{y}\|_{\infty, t}+\|v-u\|\right) .
$$

Since $b(t, x)^{\mathrm{T}} G\left(x_{t}\right) b(t, x)=I_{E}$ and $x \mapsto G(x)$ is of class $C^{1}$, the last inequality is valid for $\left(b\left(t, \widetilde{x_{n}}\right)\right)^{-1}$ and $\left(b\left(t, \widetilde{y_{n}}\right)\right)^{-1}$ instead of $b_{n}(t, x,$.$) and b_{n}(t, y,$.$) . Using$ expressions defining $\nu_{n}$ and $\sigma_{n}$ in terms of $b_{n}, b_{n}^{-1}, \nu^{\prime}$ and $\sigma^{\prime}$, the assumptions 2.4, 2.3 and inequality $\left\|b_{n}(t, x, v)\right\| \leqslant C_{\varphi n}$ we obtain easily the Lipschitz continuity of $\nu_{n}$ and $\sigma_{n}$.

Lemma 3.10. For all $(t, x) \in \Delta(E), 0 \leqslant s \leqslant t$ and $u \in E$, the following estimates hold:

$$
\left.\begin{array}{c}
\left\|b_{n}\left(s, x_{/[0, s]}, u\right)-b_{n}(t, x, u)\right\| \leqslant C_{\varphi n}|t-s|, \\
\left\|b(s, x /[0, s])^{-1} u-b(t, x)^{-1} u\right\| \leqslant C_{\varphi n}|t-s|, \\
\left\|\nu_{n}\left(s, x_{/[0, s]}, u\right)-\nu_{n}(t, x, u)\right\| \leqslant C_{\varphi n} \ell(|t-s|), \\
\left\|\sigma_{n}\left(s, x_{/[0, s]}, u\right)-\sigma_{n}(t, x, u)\right\| \leqslant C_{\varphi n} \ell(|t-s|) .
\end{array}\right\}
$$

Proof. With the notation and intermediary results of lemma 3.9, we can write

$$
\begin{aligned}
\left\|b_{n}(s, x /[0, s], u)-b_{n}(t, x, u)\right\| & =\left\|\left(b\left(s, \widetilde{x_{n} /[0, s]}\right)-b\left(t, \widetilde{x_{n}}\right)\right) \cdot \operatorname{proj}_{\overline{\mathcal{B}}(0, n)} u\right\| \\
& \leqslant n\left\|b\left(s, \widetilde{x_{n} /[0, s]}\right)-b\left(t, \widetilde{x_{n}}\right)\right\|=n\left\|E_{n}(s)-E_{n}(t)\right\| \\
& \leqslant C_{\varphi n}|t-s|\left\|\dot{\tilde{x}_{n}}\right\|_{\infty, t} \leqslant C_{\varphi n}|t-s| .
\end{aligned}
$$

Using this last inequality and the assumption 2.4 we obtain the result. 
Lemma 3.11. Let $(T, x) \in \Delta(E)$. Then there exists $\lambda_{0}>0$ such that $\alpha^{\lambda}: u \mapsto$ $\left(I+\lambda A_{n}(u, x /[0, u], .)\right)^{-1}(z)$ is continuous on $[0, T]$ for any $0<\lambda \leqslant \lambda_{0}, z \in E$.

Proof. For $(t, s) \in[0, T]^{2}$, we can write

$$
\frac{z-\alpha_{t}^{\lambda}}{\lambda} \in A_{n}\left(t, x_{/[0, t]}, \alpha_{t}^{\lambda}\right) \text { and } \quad \frac{z-\alpha_{s}^{\lambda}}{\lambda} \in A_{n}\left(s, x_{/[0, s]}, \alpha_{s}^{\lambda}\right) .
$$

Using the monotonicity, we have then

$$
\begin{aligned}
\left\|\alpha_{t}^{\lambda}-\alpha_{s}^{\lambda}\right\|^{2}= & \lambda\left(\alpha_{t}^{\lambda}-\alpha_{s}^{\lambda}, \frac{\alpha_{t}^{\lambda}-z}{\lambda}+\frac{z-\alpha_{s}^{\lambda}}{\lambda}\right) \\
= & 2 \lambda\left(\alpha_{t}^{\lambda}-\frac{\alpha_{t}^{\lambda}+\alpha_{s}^{\lambda}}{2}, \frac{\alpha_{t}^{\lambda}-z}{\lambda}+\beta_{1}\right)-\lambda\left(\alpha_{t}^{\lambda}-\alpha_{s}^{\lambda}, \beta_{1}-\beta_{2}\right) \\
& \quad+2 \lambda\left(\frac{\alpha_{s}^{\lambda}+\alpha_{t}^{\lambda}}{2}-\alpha_{s}^{\lambda}, \beta_{2}-\frac{z-\alpha_{s}^{\lambda}}{\lambda}\right) \\
\leqslant & \lambda\left(\alpha_{t}^{\lambda}-\alpha_{s}^{\lambda}, \beta_{2}-\beta_{1}\right) \leqslant \frac{1}{2} \lambda\left\|\alpha_{t}^{\lambda}-\alpha_{s}^{\lambda}\right\|^{2}+\frac{1}{2} \lambda\left\|\beta_{1}-\beta_{2}\right\|^{2}
\end{aligned}
$$

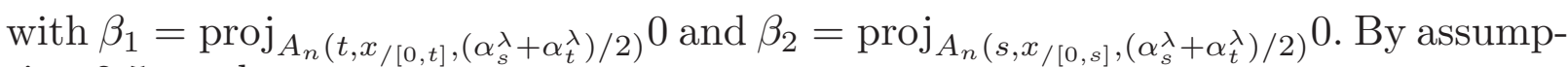
tion 2.5 , we have

$$
\left\|\alpha_{t}^{\lambda}-\alpha_{s}^{\lambda}\right\|^{2} \leqslant \lambda C_{\varphi n}\left(\ell^{2}(|t-s|)+\left|\alpha_{t}^{\lambda}-\alpha_{s}^{\lambda}\right|^{2}+\left\|x_{t}-x_{s}\right\|^{2}\right)
$$

and it is possible to choose $\lambda_{0}(\varphi, n)>0$ such that for all $\lambda \leqslant \lambda_{0}, \alpha^{\lambda}$ is continuous on $[0, T]$.

Remark 3.12. We note that $\lambda_{0}$ depends only on $n$ and $\varphi$.

Lemma 3.13. Let $r$ and $r^{\prime}$ be continuous functions from $[0, t]$ into $[0, T]$, where $t$ verifies $0 \leqslant t \leqslant T$. If we set $t_{r}=\sup _{0 \leqslant s \leqslant t} r(s)$ and $t_{r^{\prime}}=\sup _{0 \leqslant s \leqslant t} r^{\prime}(s)$, we consider $\left(t_{r}, x\right)$ and $\left(t_{r^{\prime}}, x^{\prime}\right)$ elements of $\Delta(E)$. Let us assume the existence of continuous functions $v, v^{\prime}, K$ and $K^{\prime}$ from $[0, t]$ into $E$ such that

(i) $K$ and $K^{\prime}$ are functions with bounded variations on $[0, t]$;

(ii) $\mathrm{d} K_{s} \in A_{n}\left(r(s), x_{/[0, r(s)]}, v(s)\right) \mathrm{d} s$ and $\mathrm{d} K_{s}^{\prime} \in A_{n}\left(r^{\prime}(s), x_{/\left[0, r^{\prime}(s)\right]}^{\prime}, v^{\prime}(s)\right) \mathrm{d} s$, that is, the measures

$$
\left(v_{s}-\alpha_{s}, \mathrm{~d} K_{s}-\beta_{s} \mathrm{~d} s\right)
$$

and

$$
\left(v_{s}^{\prime}-\alpha_{s}^{\prime}, \mathrm{d} K_{s}^{\prime}-\beta_{s}^{\prime} \mathrm{d} s\right)
$$

are non-negative on $[0, t]$ for any choice of continuous functions $\alpha, \alpha^{\prime}, \beta, \beta^{\prime}$ : $[0, t] \rightarrow E$ verifying

$$
\forall s \in[0, t],\left(\alpha_{s}, \beta_{s}\right) \in \operatorname{Gr}\left(A_{n}\left(r(s), x_{/[0, r(s)]}\right)\right)
$$

and

$$
\left(\alpha_{s}^{\prime}, \beta_{s}^{\prime}\right) \in G r\left(A_{n}\left(r^{\prime}(s), x_{/\left[0, r^{\prime}(s)\right]}^{\prime}\right)\right)
$$


where $\operatorname{Gr}(B)$ denotes the graph of the multivalued operator $B$ defined on the whole space $E$, i.e. the set $\left\{(x, y) \in E^{2}, y \in B(x)\right\}$. Then there exists a map $C_{\varphi n}:[0, t] \rightarrow \mathbb{R}_{+}$such that the measure

$$
\begin{aligned}
\left(v_{s}^{\prime}-v_{s}, \mathrm{~d} K_{s}^{\prime}-\mathrm{d} K_{s}\right) & +C_{\varphi n}(s)\left(\left\|x-x^{\prime}\right\|_{\infty, r(s) \wedge r^{\prime}(s)}^{2}+\left\|v-v^{\prime}\right\|_{\infty, s}^{2}\right. \\
& \left.+\left\|x(r(s))-x^{\prime}\left(r^{\prime}(s)\right)\right\|^{2}+\ell^{2}\left(\left|r(s)-r^{\prime}(s)\right|\right)\right) \mathrm{d} s
\end{aligned}
$$

is non-negative on $[0, t]$.

Remark 3.14. In the case where $A_{n}$ is independent of $t$ and $x$, the first term in (3.17) is already non-negative; the extra terms account for the dependency on $t$ and $x$.

Proof. For $p \in \mathbb{N}^{*}$ and $s \in[0, t]$, let us define

$$
\begin{aligned}
\alpha_{p}(s) & =\left(I+A_{n}(r(s), x /[0, r(s)]) / p\right)^{-1}\left(\left(v(s)+v^{\prime}(s)\right) / 2\right), \\
\beta_{p}(s) & =p\left(\left(v(s)+v^{\prime}(s)\right) / 2-\alpha_{p}(s)\right), \\
\alpha_{p}^{\prime}(s) & =\left(I+A_{n}\left(r^{\prime}(s), x^{\prime}\left[\left[0, r^{\prime}(s)\right]\right) / p\right)^{-1}\left(\left(v(s)+v^{\prime}(s)\right) / 2\right),\right.
\end{aligned}
$$

and

$$
\beta_{p}^{\prime}(s)=p\left(\left(v(s)+v^{\prime}(s)\right) / 2-\alpha_{p}^{\prime}(s)\right) .
$$

If $c:[0, t] \rightarrow E$ is continuous, the function

$$
\gamma_{p}: s \mapsto\left(I+1 / p A_{n}\left(r(s), x_{/[0, r(s)]}\right)\right)^{-1}(c(s))
$$

is continuous for $p \geqslant p_{0}$, where $p_{0}$ depends on $n$ and $\varphi$. This results from lemma 3.11. We can conclude that $\alpha_{p}, \alpha_{p}^{\prime}, \beta_{p}$ and $\beta_{p}^{\prime}$ are continuous on $[0, t]$.

By hypothesis (ii) we can write

$$
\left(v(s)-\alpha_{p}(s), \mathrm{d} K(s)-\beta_{p}(s) \mathrm{d} s\right) \geqslant 0
$$

and

$$
\left(v^{\prime}(s)-\alpha_{p}^{\prime}(s), \mathrm{d} K^{\prime}(s)-\beta_{p}^{\prime}(s) \mathrm{d} s\right) \geqslant 0 .
$$

By summation of these last inequalities, we have

$$
\begin{aligned}
\left(v(s)-\alpha_{p}(s), \mathrm{d} K(s)\right) & +\left(v^{\prime}(s)-\alpha_{p}^{\prime}(s), \mathrm{d} K^{\prime}(s)\right) \\
& -\left(v(s)-\alpha_{p}(s), \beta_{p}(s) \mathrm{d} s\right)-\left(v^{\prime}(s)-\alpha_{p}^{\prime}(s), \beta_{p}^{\prime}(s) \mathrm{d} s\right) \geqslant 0 .
\end{aligned}
$$

By results of Brézis (1973),

$$
\begin{aligned}
\lim _{p \rightarrow+\infty} \alpha_{p}(s) & =\lim _{p \rightarrow+\infty} \alpha_{p}^{\prime}(s)=\frac{1}{2}\left(v(s)+v^{\prime}(s)\right), \\
\lim _{p \rightarrow+\infty} \beta_{p}(s) & =A_{n}^{0}\left(r(s), x /[0, r(s)], \frac{1}{2}\left(v(s)+v^{\prime}(s)\right)\right)
\end{aligned}
$$

and

$$
\lim _{p \rightarrow+\infty} \beta_{p}^{\prime}(s)=A_{n}^{0}\left(r^{\prime}(s), x_{/\left[0, r^{\prime}(s)\right]}^{\prime}, \frac{1}{2}\left(v(s)+v^{\prime}(s)\right)\right),
$$


where $A_{n}^{0}\left(r(s), x_{/[0, r(s)]}\right)$ (respectively, $A_{n}^{0}\left(r^{\prime}(s), x^{\prime} /\left[0, r^{\prime}(s)\right]\right)$ ) denotes the principal section of $A_{n}\left(r(s), x_{/[0, r(s)]}\right)$ (respectively, of $A_{n}\left(r^{\prime}(t), x_{/\left[0, r^{\prime}(t)\right]}^{\prime}\right)$ ). Inequality (3.18) implies then

$$
\begin{aligned}
& \left(v(s)-v^{\prime}(s), \mathrm{d} K^{\prime}(s)-\mathrm{d} K(s)\right) \\
& \leqslant\left(v(s)-v^{\prime}(s), A_{n}^{0}\left(r(s), x_{/[0, r(s)]}, \frac{1}{2}\left(v(s)+v^{\prime}(s)\right)\right)\right. \\
& \left.-A_{n}^{0}\left(r^{\prime}(s), x_{/\left[0, r^{\prime}(s)\right]}^{\prime}, \frac{1}{2}\left(v(s)+v^{\prime}(s)\right)\right)\right) \\
& \leqslant \frac{1}{2}\left\|v(s)-v^{\prime}(s)\right\|^{2} \\
& +\frac{1}{2}\left\|A_{n}^{0}\left(r(s), x_{/[0, r(s)]}, \frac{1}{2}\left(v(s)+v^{\prime}(s)\right)\right)-A_{n}^{0}\left(r^{\prime}(s), x_{/\left[0, r^{\prime}(s)\right]}^{\prime}, \frac{1}{2}\left(v(s)+v^{\prime}(s)\right)\right)\right\|^{2} .
\end{aligned}
$$

This last inequality and assumption 2.5 yields the result.

\section{Existence of solutions in $E$}

In order to obtain the existence of a solution we will use the results of Cépa (1998), which ensure the existence of solutions to the MSDE of the following type:

$$
\mathrm{d} X_{t}+B\left(X_{t}\right) \mathrm{d} t \ni \alpha\left(X_{t}\right) \mathrm{d} t+\beta\left(X_{t}\right) \mathrm{d} W_{t},
$$

where $B$ is a maximal monotone operator on $E$ and $\alpha$ and $\beta$ verify the classical Lipschitz and linear growth conditions. If the probability space is $\left(\Omega, \mathcal{G}, \mathcal{G}_{0 \leqslant t \leqslant T}, \mathbb{Q}\right)$ and the initial condition is $\mathcal{G}_{0}$-measurable, then the solution is $\mathcal{G}_{t}$-adapted and belongs to $L^{2}\left(\Omega, C^{0}([0, T], E)\right)$.

Remark 4.1. It is crucial for the following to remark that the results of Cépa (1998) can be extended to random operator $B$ and random maps $\alpha$ and $\beta$ for which we have the following properties.

(i) There exists a constant $C>0$ such that for all $x \in E$ and $y \in E$,

$$
\begin{gathered}
\|\alpha(x)\|+\|\beta(x)\| \leqslant C(1+\|x\|) \text { a.s., } \\
\|\alpha(x)-\alpha(y)\|+\|\beta(x)-\beta(y)\| \leqslant C\|x-y\| \text { a.s. }
\end{gathered}
$$

(ii) There exists a constant $R>0$ and $M>0$ such that

$$
\forall x \in E, \quad\|x\| \leqslant R \Longrightarrow(\forall y \in B(x),\|y\| \leqslant M) \text { a.s. }
$$

(iii) The maps $\alpha$ and $\beta$ are, respectively, $\mathcal{G}_{0} \otimes \mathcal{B}(E), \mathcal{B}(E)$-measurable and $\mathcal{G}_{0} \otimes$ $\mathcal{B}(E), \mathcal{B}\left(E^{d}\right)$-measurable, where $\mathcal{B}(\mathcal{O})$ denotes the Borel $\sigma$-algebra of the topological set $\mathcal{O}$.

(iv) For all maps $Y, Z: \Omega \rightarrow E$ such that $Z \in B(Y)$ almost surely, if $Y$ is $\mathcal{G}_{0^{-}}$ measurable, then $Z$ is $\mathcal{G}_{0}$-measurable.

We give here a lemma which will be useful.

Lemma 4.2. For all $c \in E, t \in[0, T]$ and continuous map $v:[0, t] \rightarrow E$ there exists a unique $C^{1}$ map $x:[0, t] \rightarrow E$ such that

$$
\left\{\begin{array}{l}
\dot{x}(s)=b(s, x /[0, s], v(s)), \\
x(0)=c .
\end{array}\right.
$$


Proof. The space $Z=C^{1}([0, t], E)$ is a Banach space when equipped with the norm $\|f\|=\|f\|+\|\mathrm{d} f / \mathrm{d} t\|$. For $f \in Z$, let $L(f) \in Z$ be defined by $L(f)(s)=$ $c+\int_{0}^{s} b\left(u, f_{/[0, u]}, v(u)\right) \mathrm{d} u$. Using lemma $3.9, L^{m}$ is strictly contracting for large enough $m$. We infer the existence of the solution on $[0, t]$ by application of the Banach fixed point theorem on $Z$.

The construction of a solution is performed in several steps. We replace first the $v$ equation by an equation which contains only the initial data, and we can integrate it with the help of Cépa's results. We then integrate the $x$ equation according to lemma 4.2. This gives an approximate solution on an interval $\left[0, t_{1}\right]$. Assuming this interval to be small, we restart the process: we integrate an approximate $v$ equation on $\left[t_{1}, t_{2}\right]$ by using only the $x$ data from the previous interval, which is possible thanks to Cépa's results. The process is recursive and yields an approximate solution on $[0, T]$, the approximation parameter being the size of the subintervals. We take this size to be $T / 2^{p}$, and we compare the approximate solutions for the integers $p$ and $q$, and we show by stochastic techniques that the expectation of appropriate norms of the difference tends to 0 .

Theorem 4.3. For all $n \geqslant 1$, there exists a solution to the following system

$$
\left.\begin{array}{l}
\delta x_{t}^{n}=b_{n}\left(t, x_{/[0, t]}^{n}, v_{t}^{n}\right) \mathrm{d} t \\
\delta v_{t}^{n}+A_{n}\left(t, x_{/[0, t]}^{n}, v_{t}^{n}\right) \mathrm{d} t \\
\quad \ni \nu_{n}\left(t, x_{/[0, t]}^{n}, v_{t}^{n}\right) \mathrm{d} t+\sigma\left(t, x_{/[0, t]}^{n}, v_{t}^{n}\right) \delta W_{t}, \quad 0 \leqslant t \leqslant T, \\
(x(0), v(0))=\left(\eta_{1}, \eta_{2}\right) .
\end{array}\right\}
$$

Proof. To lighten the notations, we denote $b_{n}, \nu_{n}, \sigma_{n}$ and $A_{n}$ by $b, \nu, \sigma$ and $A$.

Step 1 (Construction of the approximate solution). We denote by $s^{i}(t, \xi, v)$ the square of the norm of the $i$ th row (considered as an $E$-valued vector) of $\sigma(t, \xi, v)$ for all $(t, \xi) \in \Delta(E)$ and $v \in E$. We define then

$$
\alpha(t, \xi, v)=\nu(t, \xi, v)+\frac{\partial}{\partial v}\left\{\left(s^{1}(t, \xi, v), \ldots, s^{d}(t, \xi, v)\right)^{\mathrm{T}}\right\}
$$

We consider now, for $p \in \mathbb{N}^{*}$, the uniform partition $0<t_{1}^{p}<\cdots<t_{2^{p}}^{p}=T$ of $[0, T]$ with step size $T / 2^{p}$ and we construct two processes $x^{p}$ and $v^{p}$ as follows. To simplify the notation we denote $t_{i}^{p}$ by $t_{i}$. Results of Cépa mentioned in the beginning of the section, remarks 4.1 and 2.6, (3.14), (3.16), assumptions 2.2 and 2.3 give a solution $v^{p}$ of the Itô MSDE on $\left[0, t_{1}\right]$ :

$$
\left.\begin{array}{l}
\mathrm{d} v_{t}^{p}+A\left(0, \eta_{1}, v_{t}^{p}\right) \mathrm{d} t \ni \alpha\left(0, \eta_{1}, v_{t}^{p}\right) \mathrm{d} t+\sigma\left(0, \eta_{1}, v_{t}^{p}\right) \mathrm{d} W_{t}, \\
v^{p}(0)=\eta_{2} .
\end{array}\right\}
$$

By definition of $\alpha$ and using the relation between the Itô integral and the Stratonovich integral, equation (4.3) is equivalent to the following Stratonovich MSDE

$$
\left.\begin{array}{l}
\delta v_{t}^{p}+A\left(0, \eta_{1}, v_{t}^{p}\right) \mathrm{d} t \ni \nu\left(0, \eta_{1}, v_{t}^{p}\right) \mathrm{d} t+\sigma\left(0, \eta_{1}, v_{t}^{p}\right) \delta W_{t}, \\
v^{p}(0)=\eta_{2} .
\end{array}\right\}
$$


Using lemma 4.2 , let $x^{p}$ be the solution on $\left[0, t_{1}\right]$ to

$$
\left\{\begin{array}{l}
\dot{x}^{p}(s)=b\left(s, x_{/[0, s]}^{p}, v^{p}(s)\right), \\
x^{p}(0)=\eta_{1}
\end{array}\right.
$$

Suppose $\left(x^{p}, v^{p}\right)$ defined on $\left[0, t_{1}\right] \cup \cdots \cup\left[t_{i-1}, t_{i}\right]=\left[0, t_{i}\right]$ and consider the solution $\left(v_{t}^{p}\right)_{t_{i} \leqslant t \leqslant t_{i+1}}$ on $\left[t_{i}, t_{i+1}\right]$ of the MSDE

$$
\left\{\begin{array}{l}
\delta v_{t}^{p}+A\left(t_{i}, x_{/\left[0, t_{i}\right]}^{p}, v_{t}^{p}\right) \mathrm{d} t \ni \nu\left(t_{i}, x_{/\left[0, t_{i}\right]}^{p}, v_{t}^{p}\right) \mathrm{d} t+\sigma\left(t_{i}, x_{/\left[0, t_{i}\right]}^{p}, v_{t}^{p}\right) \delta W_{t}, \\
v^{p}\left(t_{i}^{+}\right)=v^{p}\left(t_{i}^{-}\right),
\end{array}\right.
$$

and $x^{p}$ the solution on $\left[t_{i}, t_{i+1}\right]$ to

$$
\left.\begin{array}{rl}
\dot{x}^{p}(s) & =b\left(s, x_{/[0, s]}^{p}, v_{s}^{p}\right) \\
x^{p}\left(t_{i}^{+}\right) & =x^{p}\left(t_{i}^{-}\right) .
\end{array}\right\}
$$

Since $\left(v_{t}^{p}\right)_{0 \leqslant t \leqslant T}$ belongs to $L^{2}\left(\Omega, C^{0}([0, T], E)\right)$ and $\|b(s, f)\| \leqslant C_{\varphi}$ for all $(s, f) \in$ $\Delta(E),\left(x_{t}^{p}\right)_{0 \leqslant t \leqslant T}$ belongs to $L^{2}\left(\Omega, C^{1}([0, T], E)\right)$.

Step 2 (the approximate solutions form a Cauchy sequence). We will now show that $\left(x_{t}^{p}, v_{t}^{p}\right)_{0 \leqslant t \leqslant T}$ is a Cauchy sequence of $L^{2}\left(\Omega, C^{0}([0, T], E) \times C^{1}([0, T], E)\right)$. Let $1 \leqslant p \leqslant q$ be integers and denote by $0=t_{0}<t_{1}<\cdots<t_{2^{q-p}}<\cdots<$ $t_{2^{q-p+1}}<\cdots<t_{2^{q}}=T$ the partition of $[0, T]$ associated to $q$. We note that $0<$ $t_{2^{q-p}}<t_{2.2^{q-p}}<\cdots<t_{k .2^{q-p}}<\cdots<t_{2^{q}}=T$ is the partition associated with $p$. Let $s \in[0, T]$ and put $r_{1}(s)=t_{\left\lfloor s 2^{q}\right\rfloor}$ and $r_{2}(s)=t_{\left\lfloor s 2^{p}\right\rfloor}$. We then have

$$
\begin{aligned}
v_{s}^{q}-v_{s}^{p}=\int_{0}^{s}\left(\nu\left(r_{1}(u), x_{/\left[0, r_{1}(u)\right]}^{q}, v_{u}^{q}\right)-\nu\left(r_{2}(u), x_{/\left[0, r_{2}(u)\right]}^{p}, v_{u}^{p}\right)\right) \mathrm{d} u \\
\quad+\int_{0}^{s}\left(\sigma\left(r_{1}(u), x_{/\left[0, r_{1}(u)\right]}^{q}, v_{u}^{q}\right)-\sigma\left(r_{2}(u), x_{/\left[0, r_{2}(u)\right]}^{p}, v_{u}^{p}\right)\right) \delta W_{u} \\
\quad+K_{s}^{p}-K_{s}^{q}
\end{aligned}
$$

with

$$
\mathrm{d} K_{u}^{q} \in A\left(r_{1}(u), x_{/\left[0, r_{1}(u)\right]}^{q}, v_{u}^{q}\right) \mathrm{d} u, \quad 0 \leqslant u \leqslant s,
$$

and

$$
\mathrm{d} K_{u}^{p} \in A\left(r_{2}(u), x_{/\left[0, r_{2}(u)\right]}^{p}, v_{u}^{p}\right) \mathrm{d} u, \quad 0 \leqslant u \leqslant s .
$$

Using Itô's formula, we can write

$$
\begin{aligned}
\left\|v_{s}^{q}-v_{s}^{p}\right\|^{2}= & 2 \int_{0}^{s}\left(v_{u}^{q}-v_{u}^{p}, \delta v_{u}^{q}-\delta v_{u}^{p}\right) \\
= & 2 \int_{0}^{s}\left(\alpha\left(r_{1}(u), x_{/\left[0, r_{1}(u)\right]}^{q}, v_{u}^{q}\right)-\alpha\left(r_{2}(u), x_{/\left[0, r_{2}(u)\right]}^{p}, v_{u}^{p}\right), v_{u}^{q}-v_{u}^{p}\right) \mathrm{d} u \\
& +2 \int_{0}^{s}\left\|\sigma\left(r_{1}(u), x_{/\left[0, r_{1}(u)\right]}^{q}, v_{u}^{q}\right)-\sigma\left(r_{2}(u), x_{/\left[0, r_{2}(u)\right]}^{p}, v_{u}^{p}\right)\right\|^{2} \mathrm{~d} u \\
& +2 \int_{0}^{s}\left(\sigma\left(r_{1}(u), x_{/\left[0, r_{1}(u)\right]}^{q}, v_{u}^{q}\right)-\sigma\left(r_{2}(u), x_{/\left[0, r_{2}(u)\right]}^{p}, v_{u}^{p}\right), v_{u}^{q}-v_{u}^{p}\right) \mathrm{d} W_{u} \\
& +2 \int_{0}^{s}\left(v_{u}^{q}-v_{u}^{p}, \mathrm{~d} K_{u}^{p}-\mathrm{d} K_{u}^{q}\right),
\end{aligned}
$$


where the norm of matrices is defined by $\|B\|^{2}=\operatorname{tr}\left\{B^{\mathrm{T}} B\right\}$. Using lemmas 3.10, 3.9, the assumptions 2.2 and 2.3, Cauchy-Schwarz's and Burkholder-Gundy-Davis's inequalities and denoting $r(p, q)=\sup _{0 \leqslant s \leqslant T}\left|r_{1}(s)-r_{2}(s)\right|$, which tends to zero when $p$ and $q$ tend to infinity, we have

$$
\begin{aligned}
& \mathbb{E} \sup _{0 \leqslant s \leqslant t}\left\|v_{s}^{q}-v_{s}^{p}\right\|^{2} \\
& \leqslant C_{\varphi n} \ell^{2}(r(p, q))+C_{\varphi n} \int_{0}^{t}\left(\mathbb{E}\left\|x^{p}-x^{q}\right\|_{\infty, s}^{2}+\mathbb{E}\left\|\dot{x}^{p}-\dot{x}^{q}\right\|_{\infty, s}^{2}+\mathbb{E}\left\|v^{p}-v^{q}\right\|_{\infty, s}^{2}\right) \mathrm{d} s \\
& \quad+C_{\varphi n} \mathbb{E} \sqrt{\int_{0}^{t}\left[\ell^{2}(r(p, q))+\left\|x^{p}-x^{q}\right\|_{\infty, s}^{2}+\left\|\dot{x}^{p}-\dot{x}^{q}\right\|_{\infty, s}^{2}+\left\|v^{p}-v^{q}\right\|_{\infty, s}^{2}\right]\left\|v_{s}^{q}-v_{s}^{p}\right\|^{2} \mathrm{~d} s} \\
& \quad+2 \mathbb{E} \sup _{0 \leqslant s \leqslant t} \sum_{i=1}^{\left\lfloor s 2^{q}\right\rfloor} \int_{t_{i-1}}^{t_{i}}\left(v_{u}^{q}-v_{u}^{p}, \mathrm{~d} K_{u}^{p}-\mathrm{d} K_{u}^{q}\right)+\int_{\left.t_{\lfloor s 2}\right\rfloor}^{t}\left(v_{u}^{q}-v_{u}^{p}, \mathrm{~d} K_{u}^{p}-\mathrm{d} K_{u}^{q}\right) .
\end{aligned}
$$

Using the inequalities

$$
\sqrt{a+b} \leqslant \sqrt{a}+\sqrt{b}, \quad a b \leqslant(1 / \delta) a^{2}+\delta b^{2} \quad(\delta>0)
$$

and lemma 3.13 for the intervals $\left[t_{i-1}, t_{i}\right]$ (on each of them we put $r=r_{1}$ and $r^{\prime}=r_{2}$ ), we have

$$
\begin{aligned}
\mathbb{E} \sup _{0 \leqslant s \leqslant t}\left\|v_{s}^{q}-v_{s}^{p}\right\|^{2} & \\
\leqslant & C_{\varphi n} \ell^{2}(r(p, q))+C_{\varphi n} \delta \mathbb{E} \sup _{0 \leqslant s \leqslant t}\left\|v_{s}^{q}-v_{s}^{p}\right\|^{2} \\
& +\frac{C_{\varphi n}}{\delta} \int_{0}^{t}\left(\mathbb{E}\left\|x^{p}-x^{q}\right\|_{\infty, s}^{2}+\mathbb{E}\left\|\dot{x}^{p}-\dot{x}^{q}\right\|_{\infty, s}^{2}+\mathbb{E}\left\|v^{p}-v^{q}\right\|_{\infty, s}^{2}\right) \mathrm{d} s \\
+ & C_{\varphi n} \mathbb{E} \sup _{0 \leqslant s \leqslant t}\left(\sum _ { i = 1 } ^ { \lfloor s 2 ^ { q } \rfloor } \int _ { t _ { i - 1 } } ^ { t _ { i } } \left(\ell^{2}(r(p, q))+\left\|x^{p}-x^{q}\right\|_{\infty, u}^{2}\right.\right. \\
& +\int_{\left.t_{\lfloor s 2}\right\rfloor}^{t}\left(\ell^{2}(r(p, q))+\left\|\dot{x}^{p}-\dot{x}^{q}\right\|_{\infty, u}^{2}+\left\|v^{p}-v^{q}\right\|_{\infty, u}^{2}\right) \mathrm{d} u \\
+ & \left.\left.+\left\|\dot{x}^{p}-\dot{x}^{q}\right\|_{\infty, u}^{2}+\left\|v^{p}-v^{q}\right\|_{\infty, u}^{2}\right) \mathrm{~d} u\right) .
\end{aligned}
$$

Choosing $\delta$ such that $C_{\varphi n} \delta=1 / 2$, we can write

$$
\begin{aligned}
& \mathbb{E} \sup _{0 \leqslant s \leqslant t}\left\|v_{s}^{q}-v_{s}^{p}\right\|^{2} \\
& \quad \leqslant C_{\varphi n} \ell^{2}(r(p, q))+C_{\varphi n} \int_{0}^{t}\left(\mathbb{E}\left\|x^{p}-x^{q}\right\|_{\infty, s}^{2}+\mathbb{E}\left\|\dot{x}^{p}-\dot{x}^{q}\right\|_{\infty, s}^{2}+\mathbb{E}\left\|v^{p}-v^{q}\right\|_{\infty, s}^{2}\right) \mathrm{d} s .
\end{aligned}
$$


Moreover, by lemmas 3.10 and 3.9, we have

$$
\begin{aligned}
& \mathbb{E} \sup _{0 \leqslant s \leqslant t}\left\|\dot{x}_{s}^{q}-\dot{x}_{s}^{p}\right\|^{2} \\
& \quad \leqslant C_{\varphi n} \ell^{2}(r(p, q))+C_{\varphi n} \int_{0}^{t}\left(\mathbb{E}\left\|x^{p}-x^{q}\right\|_{\infty, s}^{2}+\mathbb{E}\left\|\dot{x}^{p}-\dot{x}^{q}\right\|_{\infty, s}^{2}+\mathbb{E}\left\|v^{p}-v^{q}\right\|_{\infty, s}^{2}\right) \mathrm{d} s
\end{aligned}
$$

and

$$
\begin{aligned}
& \mathbb{E} \sup _{0 \leqslant s \leqslant t}\left\|x_{s}^{q}-x_{s}^{p}\right\|^{2} \\
& \quad \leqslant C_{\varphi n} \ell^{2}(r(p, q))+C_{\varphi n} \int_{0}^{t}\left(\mathbb{E}\left\|x^{p}-x^{q}\right\|_{\infty, s}^{2}+\mathbb{E}\left\|\dot{x}^{p}-\dot{x}^{q}\right\|_{\infty, s}^{2}+\mathbb{E}\left\|v^{p}-v^{q}\right\|_{\infty, s}^{2}\right) \mathrm{d} s .
\end{aligned}
$$

The inequalities (4.6)-(4.8) imply that

$$
\begin{aligned}
& \mathbb{E}\left(\left\|x^{q}-x^{p}\right\|^{2}+\left\|\dot{x}^{q}-\dot{x}^{p}\right\|_{\infty, t}^{2}+\left\|v^{q}-v^{p}\right\|^{2}\right) \\
& \leqslant C_{\varphi n} \ell^{2}(r(p, q))+C_{\varphi n} \int_{0}^{t} \mathbb{E}\left(\left\|x^{p}-x^{q}\right\|_{\infty, s}^{2}+\left\|\dot{x}^{p}-\dot{x}^{q}\right\|_{\infty, s}^{2}+\left\|v^{p}-v^{q}\right\|_{\infty, s}^{2}\right) \mathrm{d} s,
\end{aligned}
$$

and the application of Gronwall's lemma yields

$$
\mathbb{E}\left(\left\|x^{q}-x^{p}\right\|^{2}+\left\|\dot{x}^{q}-\dot{x}^{p}\right\|_{\infty, t}^{2}+\left\|v^{q}-v^{p}\right\|^{2}\right) \leqslant C_{\varphi n} \ell^{2}(r(p, q))
$$

and the sequence $\left(x^{p}, v^{p}\right)$ is a Cauchy sequence in $L^{2}\left(\Omega, C^{1}([0, T], E) \times C^{0}([0, T], E)\right)$ and there exists then a stochastic process $(x, v)$ which is the limit of $\left(x^{p}, v^{p}\right)$.

To complete the proof of existence we must prove that $(x, v)$ verifies the MSDE. For this it remains to show that $\left(K^{p}\right)_{p \geqslant 1}$ converges almost surely towards a process $\left(K_{t}\right)_{0 \leqslant t \leqslant T}$ with bounded variation on $[0, T]$ and verifying $\mathrm{d} K_{t} \in A\left(t, x_{/[0, t]}, v_{t}\right) \mathrm{d} t$. Since $\left(x^{p}, v^{p}\right)$ converges in $L^{2}\left(\Omega, C^{1}([0, T], E) \times C^{0}([0, T], E)\right)$, there exists a subsequence, which is still denoted by $p$, such that $\left(x^{p}, v^{p}\right)$ tends almost surely to $(x, v)$ in $C^{1}([0, T], E) \times C^{0}([0, T], E)$. It follows that $K^{p}$ tends almost surely to a process $K$ in $C^{0}([0, T], E)$.

Step 3 (uniform estimation of the total variation of $K$ on $[0, T]$ ). For each $p \in \mathbb{N}^{*}$, we consider the above partition $0<t_{1}^{p}<\cdots<t_{2^{p}}^{p}=T$ of $[0, T]$ and we denote by $r_{p}(t)$ the $t_{i}^{p}$ such that $t_{i}^{p} \leqslant t<t_{i+1}^{p}$.

Lemma 4.4. There exists a random variable almost surely non-negative which estimates from above for all $p$ the total variation $\left|K^{p}\right|_{\mathrm{T}}$ of $K^{p}$ on $[0, T]$.

Proof. Let $\gamma$ be a positive real and $\Lambda\left(\gamma, V^{\prime}\right)=\{(t, y, v) \in[0, T] \times T M, y \in$ $\left.V^{\prime},\|v\|_{y} \leqslant \gamma\right\}$. By remark 2.6,

$$
\mu=\sup _{(t, y, v) \in \Lambda\left(\gamma, V^{\prime}\right)}\left\|A^{\prime}(t, y, v)\right\|_{y}
$$


is finite. We apply proposition 4.4 of Cépa (1998) (with $a=0$ and $\gamma$ ) to the process $\left(v^{p}, K^{p}\right)$ on each $\left[t_{i}^{p}, t_{i+1}^{p}\right]$ to obtain for all $0 \leqslant s \leqslant t \leqslant T$

$$
\begin{aligned}
\gamma\left|K^{p}\right|_{s}^{t} \leqslant & \sum_{i=k}^{l} \gamma\left|K^{p}\right|_{t_{i}}^{t_{i+1}}+\gamma\left|K^{p}\right|_{s}^{t_{k}}+\gamma\left|K^{p}\right|_{t_{l}}^{t_{l}} \\
\leqslant & \sum_{i=k}^{l}\left\{\int_{t_{i}}^{t_{i+1}}\left(v^{p}(u), \mathrm{d} K^{p}(u)\right)\right\}+\int_{t}^{t_{l}}\left(v^{p}(u), \mathrm{d} K^{p}(u)\right) \\
& +\int_{s}^{t_{k}}\left(v^{p}(u), \mathrm{d} K^{p}(u)\right) \\
& +\sum_{i=k}^{l}\left\{\mu \int_{t_{i}}^{t_{i+1}}\left\|v^{p}(u)\right\| \mathrm{d} u+\gamma \mu\left(t_{i+1}-t_{i}\right)\right\} \\
& +\mu \int_{s}^{t_{k}}\left\|v^{p}(u)\right\| \mathrm{d} u+\gamma \mu\left(t_{k}-s\right) \\
& +\mu \int_{t_{l}}^{t}\left\|v^{p}(u)\right\| \mathrm{d} u+\gamma \mu\left(t-t_{l}\right) \\
= & \int_{s}^{t}\left(v^{p}(u), \mathrm{d} K^{p}(u)\right)+\mu \int_{s}^{t}\left\|v^{p}(u)\right\| \mathrm{d} u+\gamma \mu(t-s),
\end{aligned}
$$

where $t_{k}$ and $t_{l}$ verify $t_{k-1}<s \leqslant t_{k}$ and $t_{l} \leqslant t<t_{l+1}$. Otherwise, with $w^{p}=v^{p}+K^{p}$, we have

$$
\left\|v^{p}(t)\right\|^{2}=\left\|w^{p}(t)\right\|^{2}-2 \int_{0}^{t}\left(v^{p}(u), \mathrm{d} K^{p}(u)\right)+2 \int_{0}^{t}\left(w^{p}(u)-w^{p}(t), \mathrm{d} K^{p}(u)\right)
$$

and

$$
-\left\|v^{p}(s)\right\|^{2}=-\left\|w^{p}(s)\right\|^{2}+2 \int_{0}^{s}\left(v^{p}(u), \mathrm{d} K^{p}(u)\right)-2 \int_{0}^{s}\left(w^{p}(u)-w^{p}(s), \mathrm{d} K^{p}(u)\right) .
$$

By summation of the two last equalities and using the inequality (4.10), we can write

$$
2\left[\gamma-\sup \left\{\left\|w^{p}(u)-w^{p}(v)\right\|,|u-v| \leqslant|t-s|\right\}\right]\left|K^{p}\right|_{s}^{t} \leqslant C_{\varphi n}(\omega),
$$

since $v^{p}, K^{p}$ and $w^{p}$ are almost surely uniformly bounded with $p$ on $[0, T]$. Recalling that $\left(w^{p}\right)_{p \geqslant 1}$ converges uniformly towards a continuous function $w$ we can conclude by Ascoli's theorem that there exists $\eta_{\varphi n}(\omega)>0$ such that $\sup \left\{\left\|w^{p}(u)-w^{p}(v)\right\|, \mid u-\right.$ $\left.v \mid \leqslant \eta_{\varphi n}\right\} \leqslant \gamma / 2$ and then $\left|K^{p}\right|_{s}^{t} \leqslant C_{\varphi n}(\omega) / \gamma$. If $0=s_{0}<s_{1}<\cdots<s_{\mathrm{N}}=T$ is a partition of $[0, T]$ such that $s_{i+1}-s_{i} \leqslant \eta_{\varphi n}$, then

$$
\left|K^{p}\right|_{\mathrm{T}} \leqslant \sum_{i=0}^{N-1}\left|K^{p}\right|_{s_{i}}^{s_{i+1}} \leqslant N_{\varphi n} \frac{C_{\varphi n}(\omega)}{\gamma}
$$

This last result implies that $K$ is almost surely with bounded variation on $[0, T]$ and for all sequences of processes $\varphi_{p} \in C([0, T], E)$ converging almost surely in 
$C([0, T], E)$ to a process $\varphi$, then (see Saisho (1987) for more details):

$$
\lim _{p \rightarrow+\infty} \int_{s}^{t}\left(\varphi_{p}(u), \mathrm{d} K^{p}(u)\right)=\int_{s}^{t}(\varphi(u), \mathrm{d} K(u)), \quad 0 \leqslant s \leqslant t \leqslant T .
$$

Step 4 (end of the proof). Let $\alpha, \beta:[0, T] \rightarrow E$ be two continuous functions such that $\beta_{t}$ belongs to $A\left(t, x_{/[0, t]}, \alpha_{t}\right)$. Let us set $\beta_{p}(t)=\operatorname{proj}_{\mathcal{K}_{p}(t)} \beta_{t}$ with $\mathcal{K}_{p}(t)=$ $A\left(r_{p}(t), x_{/\left[0, r_{p}(t)\right]}^{p}, \alpha_{t}\right)$. By assumption 2.5,

$$
\left\|\beta_{p}(t)-\beta(t)\right\| \leqslant C_{\varphi n}(\omega)\left(\left\|x-x^{p}\right\|_{\infty, r_{p}(t)}+\left\|x(t)-x^{p}\left(r_{p}(t)\right)\right\|+\ell\left(\left|t-r_{p}(t)\right|\right)\right)
$$

and then $\left(\beta_{p}\right)_{p}$ converges almost surely uniformly to $\beta$. Moreover, $\beta_{p}$ is almost surely continuous on each $\left[t_{i}^{p}, t_{i+1}^{p}[\right.$ and then

$$
\int_{t_{i}^{p}}^{t_{i+1}^{p}}\left(v^{p}(u)-\alpha(u), \mathrm{d} K^{p}(u)-\beta_{p}(u) \mathrm{d} u\right) \geqslant 0 .
$$

We deduce from this last inequality, (4.11) and the dominated Lebesgue theorem that

$$
\int_{s}^{t}(v(u)-\alpha(u), \mathrm{d} K(u)-\beta(u) \mathrm{d} u) \geqslant 0, \quad 0 \leqslant s \leqslant t \leqslant T,
$$

which is the definition of $\mathrm{d} K(t) \in A\left(t, x_{/[0, t]}, v_{t}\right) \mathrm{d} t$.

\section{Existence and uniqueness in the local chart}

(a) Existence

We return to the notation $b_{n}, \nu_{n}, \sigma_{n}$ and $A_{n}$, and $n$ will tend to infinity. Let $\tau_{n}$ be the stopping time defined by $\tau_{n}(\omega)=\inf \left\{t \leqslant T,\left\|\dot{x}_{t}^{n}(\omega)\right\| \geqslant n\right.$ or $\left.\left\|v_{t}^{n}(\omega)\right\| \geqslant n\right\}$. Clearly, $\left(\tau_{n}\right)_{n \geqslant 1}$ is a non-decreasing sequence of stopping times and $\left(x_{t}^{n}, v_{t}^{n}\right)=\left(x_{t}^{m}, v_{t}^{m}\right)$ almost surely on $\left[0, \tau_{n}\right]$ for all $n \leqslant m$. We note then $\tau=\lim _{n \rightarrow+\infty} \tau_{n}$ and we put, for $0 \leqslant t<\tau(\omega), x(t, \omega)=x^{n}(t, \omega)$ and $v(t, \omega)=v^{n}(t, \omega)$, where $n$ is such that $t \leqslant \tau_{n}(\omega)$. Therefore, by theorem 4.3 and lemma $3.5,(x, v)$ verifies:

$$
\begin{aligned}
& \delta x_{t}=\tilde{b}\left(t, x /[0, t], v_{t}\right) \mathrm{d} t \\
& \delta v_{t}+\tilde{A}\left(t, x_{/[0, t]}, v_{t}\right) \mathrm{d} t \\
& \quad \ni \tilde{\nu}\left(t, x /[0, t], v_{t}\right) \mathrm{d} t+\tilde{\sigma}\left(t, x_{/[0, t]}, v_{t}\right) \delta W_{t}, \quad 0 \leqslant t<\tau, \\
& (x(0), v(0))=\left(\eta_{1}, \eta_{2}\right) .
\end{aligned}
$$

Lemma 5.1. Almost surely, $\tau=T$.

Proof. It suffices to prove for all $t \leqslant T$,

$$
\lim _{n \rightarrow+\infty} \mathbb{P}\left\{\tau_{n} \leqslant t\right\}=0 .
$$

Let $t<T, n \geqslant 1$. Since for all $0 \leqslant s \leqslant t \wedge \tau_{n}, \dot{x}_{s}=\tilde{b}\left(s, x_{/[0, s]}, v_{s}\right)$,

$$
\left\|\dot{x}_{s}\right\| \leqslant C_{\varphi}\left\|v_{s}\right\|
$$


and therefore, since $t<T$,

$$
\left\|x_{s}\right\| \leqslant\left\|\eta_{1}\right\|+C_{\varphi}\|v\|_{\infty, s} .
$$

We write

$$
\begin{aligned}
\left\|v_{s \wedge \tau_{n}}\right\|^{2}=\| & \eta_{2} \|^{2}+2 \int_{0}^{s \wedge \tau_{n}}\left(v_{u}, \delta v_{u}\right) \\
=\| & \eta_{2} \|^{2}+2 \int_{0}^{s \wedge \tau_{n}}\left(v_{u}, \alpha\left(u, x /[0, u], v_{u}\right)\right) \mathrm{d} u \\
& +2 \int_{0}^{s \wedge \tau_{n}} \operatorname{tr}\left\{\sigma\left(u, x_{/[0, u]}, v_{u}\right)^{\mathrm{T}} \sigma\left(u, x /[0, u], v_{u}\right)\right\} \mathrm{d} u \\
& -2 \int_{0}^{s \wedge \tau_{n}}\left(v_{u}, \mathrm{~d} K_{u}\right) \\
& +2 \int_{0}^{s \wedge \tau_{n}}\left(v_{u}, \sigma\left(u, x_{/[0, u]}, v_{u}\right)\right) \mathrm{d} W_{u} .
\end{aligned}
$$

Let $0 \leqslant u \leqslant v \leqslant T$. With the same notation of lemma 4.4 and by (4.10),

$$
\gamma\left|K^{p}\right|_{u}^{v} \leqslant \int_{u}^{v}\left(v^{p}(s), \mathrm{d} K^{p}(s)\right)+\mu \int_{u}^{v}\left\|v^{p}(s)\right\| \mathrm{d} s+\gamma \mu(v-u),
$$

and then, when $p$ tends to infinity,

$$
-\int_{u}^{v}(v(s), \mathrm{d} K(s)) \leqslant \mu \int_{u}^{v}\|v(s)\| \mathrm{d} s+\gamma \mu(v-u) .
$$

Lemma 3.3 gives the growth properties of $\tilde{b}, \tilde{\nu}$ and $\tilde{\sigma}$ and then, by assumption 2.3, the growth property of $\alpha$. Using this lemma, (5.4) with $u=0$ and $v=s \wedge \tau_{n}$, the Cauchy-Schwarz inequality and writing $\mu\|v(s)\| \leqslant\left(\mu^{2}+\|v(s)\|^{2}\right) / 2$, we have

$$
\begin{aligned}
\left\|v_{s \wedge \tau_{n}}\right\|^{2} \leqslant \| & \eta_{2}\left\|^{2}+C_{\varphi}+C_{\varphi} \int_{0}^{s \wedge \tau_{n}}\right\| v_{u} \|^{2} \mathrm{~d} u \\
& +C_{\varphi} \int_{0}^{s \wedge \tau_{n}}\left(1+\|x\|_{\infty, u}+\|\dot{x}\|_{\infty, u}+\|v\|_{\infty, u}\right)^{2} \mathrm{~d} u \\
& +2 \int_{0}^{s \wedge \tau_{n}}\left(v_{u}, \sigma\left(u, x /[0, u], v_{u}\right)\right) \mathrm{d} W_{u} .
\end{aligned}
$$

Taking the suprema and the expectation in the last inequality, using the assumptions 2.3 and 2.4, inequality $a b \leqslant\left((1 / \varepsilon) a^{2}+\varepsilon b^{2}\right) / 2, \varepsilon>0$, the Burkhölder-GundyDavis inequalities, (5.2) and (5.3)

$$
\begin{aligned}
\mathbb{E} \sup _{0 \leqslant s \leqslant t}\left\|v_{s \wedge \tau_{n}}\right\|^{2} \leqslant C_{\varphi} & +C_{\varphi} \int_{0}^{t} \mathbb{E} \sup _{0 \leqslant u \leqslant s}\left\|v_{u \wedge \tau_{n}}\right\|^{2} \mathrm{~d} s \\
& +C_{\varphi} \mathbb{E}^{1 / 2}\left\{\int_{0}^{t}\left\|v_{s}\right\|^{2}\left(1+\|v\|_{\infty, s}^{2}\right) \mathrm{d} s\right\}
\end{aligned}
$$

and then

$$
\mathbb{E} \sup _{0 \leqslant s \leqslant t}\left\|v_{s \wedge \tau_{n}}\right\|^{2} \leqslant C_{\varphi}+C_{\varphi} \int_{0}^{t} \mathbb{E} \sup _{0 \leqslant u \leqslant s}\left\|v_{u \wedge \tau_{n}}\right\|^{2} \mathrm{~d} s+\frac{1}{2} \mathbb{E} \sup _{0 \leqslant s \leqslant t}\left\|v_{s \wedge \tau_{n}}\right\|^{2},
$$


and the Gronwall lemma yields

$$
\mathbb{E}\left\|v_{t \wedge \tau_{n}}\right\|^{2} \leqslant C_{\varphi}
$$

and by (5.2),

$$
\mathbb{E}\left\|\dot{x}_{t \wedge \tau_{n}}\right\|^{2} \leqslant C_{\varphi}
$$

Now,

$$
\begin{aligned}
\mathbb{E}\left(\left\|v_{t \wedge \tau_{n}}\right\|^{2}+\left\|\dot{x}_{t \wedge \tau_{n}}\right\|^{2}\right) & =\int_{\Omega}\left(\left\|v_{t \wedge \tau_{n}}\right\|^{2}+\left\|\dot{x}_{t \wedge \tau_{n}}\right\|^{2}\right) \mathrm{d} \mathbb{P} \\
& \geqslant \int_{\left\{\tau_{n} \leqslant t\right\}}\left(\left\|v_{t \wedge \tau_{n}}\right\|^{2}+\left\|\dot{x}_{t \wedge \tau_{n}}\right\|^{2}\right) \mathrm{d} \mathbb{P} \\
& \geqslant n^{2} \mathbb{P}\left(\tau_{n} \leqslant t\right)
\end{aligned}
$$

and then

$$
\mathbb{P}\left(\tau_{n} \leqslant t\right) \leqslant \frac{C_{\varphi}}{n^{2}}
$$

which completes the proof.

Theorem 5.2. There exists a solution to the system

$$
\left\{\begin{array}{l}
\delta x_{t}=\tilde{b}\left(t, x /[0, t], v_{t}\right) \mathrm{d} t \\
\delta v_{t}+\tilde{A}\left(t, x /[0, t], v_{t}\right) \mathrm{d} t \ni \tilde{\nu}\left(t, x_{/[0, t]}, v_{t}\right) \mathrm{d} t+\tilde{\sigma}\left(t, x_{/[0, t]}, v_{t}\right) \delta W_{t}, \quad 0 \leqslant t<T, \\
(x(0), v(0))=\left(\eta_{1}, \eta_{2}\right) .
\end{array}\right.
$$

Proof. Since $\tau=T$, the construction of $(x, v)$ from $\left(x^{n}, v^{n}\right)$ and theorem 4.3 give the result.

By definition of $\tilde{b}, \tilde{A}, \tilde{\nu}$ and $\tilde{\sigma}$, if we put $\tau_{U}=\inf \{t<T, x(t) \notin U\} \wedge T$, $\left(x_{t}, v_{t}\right)_{0 \leqslant t<\tau_{U}}$ verifies

$$
\left\{\begin{array}{l}
\delta x_{t}=b\left(t, x /[0, t], v_{t}\right) \mathrm{d} t \\
\delta v_{t}+A\left(t, x /[0, t], v_{t}\right) \mathrm{d} t \ni \nu\left(t, x_{/[0, t]}, v_{t}\right) \mathrm{d} t+\sigma\left(t, x_{/[0, t]}, v_{t}\right) \delta W_{t}, \quad 0 \leqslant t<\tau_{U}, \\
(x(0), v(0))=\left(\eta_{1}, \eta_{2}\right) .
\end{array}\right.
$$

Remark 5.3. We can remark that the process $\left(x_{t}, v_{t}\right)_{0 \leqslant t<\tau_{U}}$ is $\mathcal{F}_{t}$-adapted.

\section{(b) Uniqueness}

We have the following proposition.

Proposition 5.4. Let $(x, v)$ and $\left(x^{\prime}, v^{\prime}\right)$ be solutions to (4.1), then $x=x^{\prime}$ almost surely and $v=v^{\prime}$ almost surely. 
Proof. Indeed, by Itô's formula,

$$
\begin{aligned}
\left\|v_{t}-v_{t}^{\prime}\right\|^{2}= & 2 \int_{0}^{t}\left(v_{s}-v_{s}^{\prime}, \delta v_{s}-\delta v_{s}^{\prime}\right) \\
= & 2 \int_{0}^{t}\left(\nu_{n}\left(s, x_{/[0, s]}, v_{s}\right)-\nu_{n}\left(s, x_{/[0, s]}^{\prime}, v_{s}^{\prime}\right), v_{s}-v_{s}^{\prime}\right) \mathrm{d} s \\
& +2 \int_{0}^{t}\left\|\sigma_{n}\left(s, x /[0, s], v_{s}\right)-\sigma_{n}\left(s, x_{/[0, s]}^{\prime}, v_{s}^{\prime}\right)\right\|^{2} \mathrm{~d} s \\
& +2 \int_{0}^{t}\left(\sigma_{n}\left(s, x /[0, s], v_{s}\right)-\sigma_{n}\left(s, x_{/[0, s]}^{\prime}, v_{s}^{\prime}\right), v_{s}-v_{s}^{\prime}\right) \delta W_{s} \\
& +2 \int_{0}^{t}\left(v_{s}-v_{s}^{\prime}, \mathrm{d} K_{s}^{\prime}-\mathrm{d} K_{s}\right) .
\end{aligned}
$$

Using lemma 3.9 , the inequality $\mathbb{E}\left\|x_{t}-x_{t}^{\prime}\right\|^{2} \leqslant C_{\varphi n} \mathbb{E}\left\|v_{t}-v_{t}^{\prime}\right\|^{2}$ and lemma 3.13, (5.5) becomes

$$
\mathbb{E} \sup _{0 \leqslant s \leqslant t}\left\|v_{s}-v_{s}^{\prime}\right\|^{2} \leqslant C_{\varphi n}(t) \int_{0}^{t} \mathbb{E} \sup _{0 \leqslant u \leqslant s}\left\|v_{u}-v_{u}^{\prime}\right\|^{2} \mathrm{~d} s,
$$

and the Gronwall lemma yields the result.

\section{(c) Independence on the chart}

Let $V_{1}^{\prime}$ and $V_{2}^{\prime}$ be two open subsets of $M$ such that $V^{\prime}=V_{1}^{\prime} \cap V_{2}^{\prime} \neq \emptyset$ and let $x_{0} \in V^{\prime}$. We denote by $E$ the tangent space of $M$ at the point $x_{0}$. Let $W^{\prime}$ be an open subset of $M$ such that $x_{0} \in W^{\prime} \subset \overline{W^{\prime}} \subset V^{\prime}$ and each pair of points of $W^{\prime}$ can be linked by a unique geodesic of $M$. Let $\varphi_{1}: V_{1}^{\prime} \subset M \rightarrow V_{1} \subset E, \psi_{1}=\varphi_{1}^{-1}, \varphi_{2}$ : $V_{2}^{\prime} \subset M \rightarrow V_{2} \subset E, \psi_{2}=\varphi_{2}^{-1}$ two local charts of $M$ such that $\varphi_{1}\left(x_{0}\right)=\varphi_{2}\left(x_{0}\right)=0$ and $T_{x_{0}} \varphi_{1}=T_{x_{0}} \varphi_{2}=I_{E}$. We denote by $b_{i}, \nu_{i}, \sigma_{i}$ and $A_{i}, 1 \leqslant i \leqslant 2$ the maps associated to $\varphi_{i}$. For all open subsets $U^{\prime}$ of $M$ verifying $\overline{W^{\prime}} \subset U^{\prime} \subset \overline{U^{\prime}} \subset V^{\prime}$ and for all second-order random variables $\eta$ on $T M$ such that $\pi(\eta) \in W^{\prime}$, we put $U=\varphi\left(U^{\prime}\right)$ and denote by $\left(x^{i}, v^{i}\right),(1 \leqslant i \leqslant 2)$, the solution to

$$
\left.\begin{array}{l}
\mathrm{d} x_{t}^{i}=b^{i}\left(t, x_{/[0, t]}^{i}, v_{t}^{i}\right) \mathrm{d} t \\
\delta v_{t}^{i}+A^{i}\left(t, x_{/[0, t]}^{i}, v_{t}^{i}\right) \mathrm{d} t \ni \nu^{i}\left(t, x_{/[0, t]}^{i}, v_{t}^{i}\right) \mathrm{d} t+\sigma^{i}\left(t, x_{/[0, t]}^{i}, v_{t}^{i}\right) \delta W_{t}, \\
\left(x^{i}(0), v^{i}(0)\right)=\left(\varphi_{i}(\eta), \bar{\tau}\left(\pi(\eta) x_{0}\right) \eta\right),
\end{array}\right\}
$$

until the exit time $\tau_{U}^{i}$ of $U$.

Let $\tau_{U}=\tau_{U}^{1} \wedge \tau_{U}^{2}$. To prove the independence on the chart, it suffices to show that $x_{t}^{2}=\varphi_{2}\left(\psi_{1}\left(x_{t}^{1}\right)\right)$ and $v_{t}^{2}=v_{t}^{1}$ for all $t<\tau_{U}$. To do this, by uniqueness of (5.6), we will prove that $\left(\varphi_{2}\left(\psi_{1}\left(x_{t}^{1}\right)\right), v_{t}^{1}\right)$ is a solution of (5.6) with $i=2$. For $t<\tau_{U}$, let us define $y_{t}=\varphi_{2}\left(\psi_{1}\left(x_{t}^{1}\right)\right)$ and write

$$
\begin{aligned}
\dot{y}(t) & =T_{\psi_{1}\left(x_{t}^{1}\right) \varphi_{2}}\left(T_{x_{t}^{1}} \psi_{1} \dot{x}_{t}^{1}\right) \\
& =\left(T_{\psi_{2}\left(x^{2}\right)(t)} \varphi_{2} \tau\left(\psi_{2}\left(x_{/[0, t]}^{2}\right)\right) \bar{\tau}\left(x_{0}, \pi(\eta)\right)\right)\left(\bar{\tau}\left(\pi(\eta), x_{0}\right)\left(\tau\left(\psi_{1}\left(x_{/[0, t]}^{1}\right)\right)\right)^{-1} T_{x_{t}^{1}} \psi_{1} \dot{x}_{t}^{1}\right) \\
& =b_{2}\left(t, x_{/[0, t]}^{2}\right)\left(b_{1}\left(t, x_{/[0, t]}^{1}\right)\right)^{-1} \dot{x}_{t}^{1} \\
& =b_{2}\left(t, x_{/[0, t]}^{2}, v_{t}^{1}\right) .
\end{aligned}
$$


To complete the proof, it remains to show that $\nu_{2}\left(t, y_{/[0, t]}\right)=\nu_{1}\left(t, x_{/[0, t]}^{1}\right)$ and similar equalities for $\sigma_{2}$ and $A_{2}$. By definition of $\nu_{2}, \nu_{1}, x^{1}$ and $x^{2}$, we can write, for $0 \leqslant t<$ $\tau_{U}$ and $v \in E$ :

$$
\begin{aligned}
\nu_{2}(t, y /[0, t], v) & =\bar{\tau}\left(\pi(\eta), x_{0}\right)\left(\tau\left(\psi_{2}(y /[0, t])\right)\right)^{-1} \nu^{\prime}\left(t, \psi_{2}(y)(t), \tau\left(\psi_{2}(y) \bar{\tau}\left(x_{0}, \pi(\eta)\right) v\right)\right. \\
& =\bar{\tau}\left(\pi(\eta), x_{0}\right)\left(\tau\left(\psi_{1}\left(x_{/[0, t]}^{1}\right)\right)\right)^{-1} \nu^{\prime}\left(t, \psi_{1}\left(x_{1}\right)(t), \tau\left(\psi_{1}\left(x_{/[0, t]}^{1}\right) \bar{\tau}\left(x_{0}, \pi(\eta)\right) v\right)\right. \\
& =\nu_{1}\left(t, x_{/[0, t]}^{1}, v\right)
\end{aligned}
$$

and the calculations are similar for $\sigma_{2}$ and $A_{2}$.

In $\S 3$, we have shown the existence and uniqueness in a local chart, and in this section we have proved that two local solutions starting with the same local initial data obtained thanks to several local charts define the same solution on the manifold.

\section{Global existence on the manifold}

We have the following result.

Proposition 6.1. Let $T$ be a strictly positive number, and let $A^{\prime}, \sigma^{\prime}$ and $\nu^{\prime}$ satisfy assumptions 2.2-2.5. Let $\mathcal{E}^{\prime}=\left(\Omega, \mathcal{F}^{\prime},\left(\mathcal{F}_{t}^{\prime}\right)_{t \geqslant 0}, \mathbb{P}\right)$ be a filtered probability space verifying the usual conditions. Let $B_{t}$ be the standard $\mathbb{R}^{d}$ Brownian motion on $\mathcal{E}^{\prime}$, and let $T_{0}$ be an $\mathcal{F}_{t}^{\prime}$-stopping time with values in $[0, T]$ almost surely. Let $\eta^{\prime}$ be an $\mathcal{F}_{T_{0}}^{\prime}$-measurable random variable which takes its values in $T M$ and whose second-order moment is finite. There exists a unique $\mathcal{F}_{t}^{\prime}$-adapted solution $\left(x_{t}^{\prime}, v_{t}^{\prime}\right)$ to the following system:

$$
\left.\begin{array}{l}
\delta x_{t}^{\prime}=v_{t}^{\prime} \mathrm{d} t, \\
D_{\dot{x}_{t}^{\prime}} v_{t}^{\prime}+A^{\prime}\left(t, x_{t}^{\prime}, v_{t}^{\prime}\right) \mathrm{d} t \ni \nu^{\prime}\left(t, x_{t}^{\prime}, v_{t}^{\prime}\right) \mathrm{d} t+\sigma^{\prime}\left(t, x_{t}^{\prime}, v_{t}^{\prime}\right) \delta B_{t}, \quad T_{0} \leqslant t \leqslant T, \\
\left(x^{\prime}\left(T_{0}\right), v^{\prime}\left(T_{0}\right)\right)=\left(\pi\left(\eta^{\prime}\right), \eta^{\prime}\right), \text { a.s. }
\end{array}\right\}
$$

Proof. We keep the notation used until now. Under the assumption that $\pi\left(\eta^{\prime}\right)$ takes its values almost surely in $W^{\prime}$, we have obtained the existence of a solution $\left(x_{t}^{\prime}, v_{t}^{\prime}\right)_{T_{0} \leqslant t<\tau_{U^{\prime}}}$ of $(2.1)$ for all open subsets $U^{\prime}$ of $M$ containing the closed set $\overline{W^{\prime}}$ and which can be described by a unique chart of $M$. Moreover, $\left(x_{S}^{\prime}, v_{S}^{\prime}\right)$ is $\mathcal{F}_{S}^{\prime}$-measurable for all $\mathcal{F}_{t}^{\prime}$-stopping time $S$ such that $T_{0} \leqslant S \leqslant T$ almost surely. We now suppose that $\eta^{\prime}$ can take its values in all $T M$.

A consequence of assumption 2.1 is that $M$ can be covered by an, at most, countable number of open sets $W_{i}^{\prime}$ such that

(i) for each pair of points of $W_{i}^{\prime}$ there is a unique geodesic between them;

(ii) $\pi^{-1} W_{i}^{\prime}$ is trivializable;

(iii) $W_{i}^{\prime}$ is included in one of the balls $B_{M}\left(z_{k}, r^{\prime}\right)$.

Let $W_{i}^{\prime \prime}$ now be a measurable partition of $M$ such that $W_{i}^{\prime \prime}$ is included in $W_{i}^{\prime}$. We define

$$
\Omega_{i}=\left(\pi \eta^{\prime}\right)^{-1} W_{i}^{\prime \prime}
$$

and $\eta_{i}^{\prime}$ a random variable on $W_{i}^{\prime \prime}$ which coincides with $\eta^{\prime}$ on $\Omega_{i}$. Let $\left(x_{i}^{\prime}, v_{i}^{\prime}\right)$ be the solution to (2.1) with initial condition $\eta_{i}^{\prime}$ at time $T_{0}$. Let $K(i)$ be the set of indices $k$ 


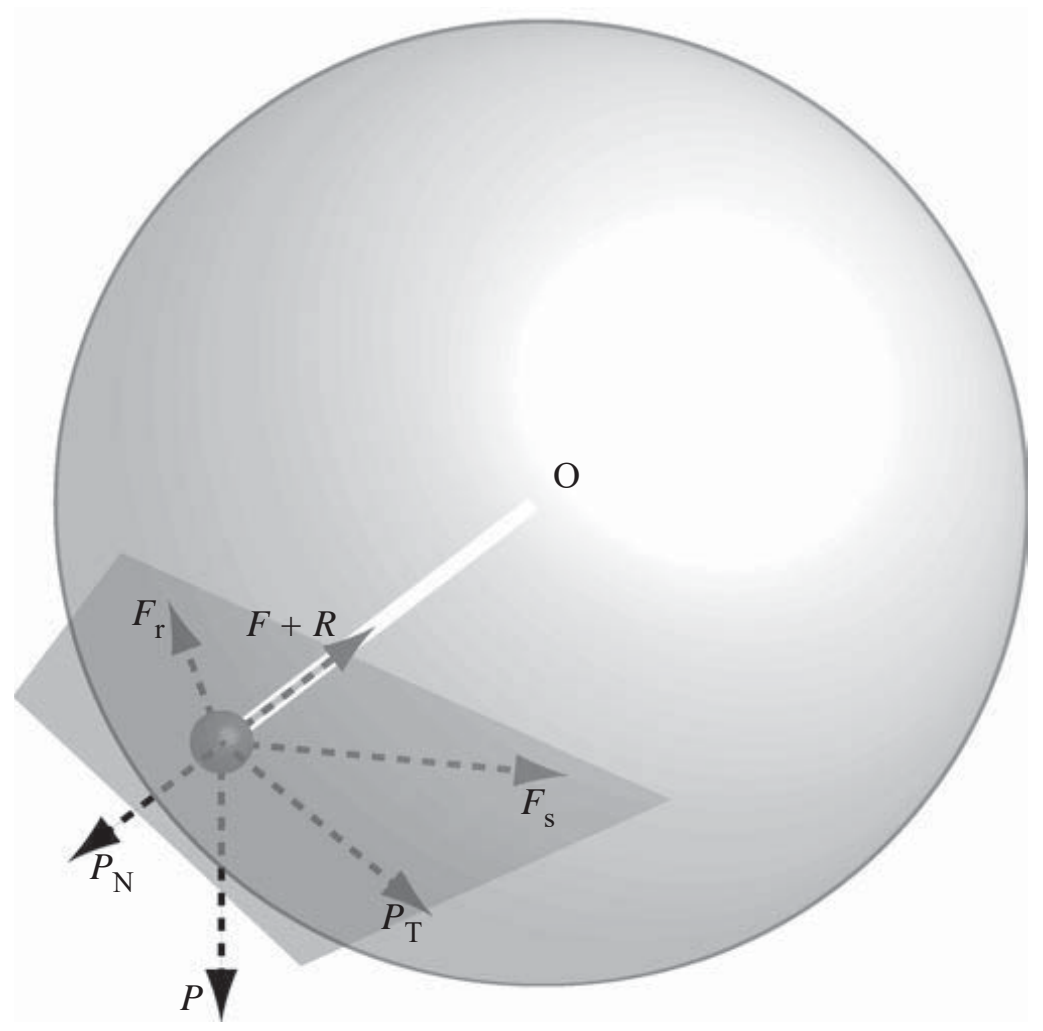

Figure 1. The weight is $P$, its normal and tangential components are, respectively, $P_{\mathrm{N}}$ and $P_{\mathrm{T}}$, $F_{\mathrm{r}}$ is the friction force, $F_{\mathrm{s}}$ is the stochastic force and $F+R$ is the sum of the reaction of the constraints and the tension of the stem.

such that $W_{i}^{\prime}$ is included in $B_{M}\left(z_{k}, r^{\prime}\right)$. There is for each $k \in K(i)$ an exit time $\sigma_{k}$, which is the largest time for which $x_{i /\left[T_{0}, \sigma_{k}[\right.}^{\prime}$ belongs to $B_{M}\left(z_{k}, r\right)$, and we let

$$
\bar{\sigma}_{i}=\max \left\{\sigma_{k}, k \in K(i)\right\} .
$$

We define now a $\mathcal{F}_{t}^{\prime}$-stopping time

$$
\tau\left(T_{0}, \eta^{\prime}\right)=\sum_{i} \mathbf{1}_{\Omega_{i}} \bar{\sigma}_{i}
$$

then $\left(x^{\prime}, v^{\prime}\right)$ is obtained by patching together the $\left(x_{i}^{\prime}, v_{i}^{\prime}\right)$ :

$$
\left(x^{\prime}, v^{\prime}\right)=\sum_{i} \mathbf{1}_{\Omega_{i}}\left(x_{i}^{\prime}, v_{i}^{\prime}\right),
$$

which is well defined for all stopping times $S \in\left[T_{0}, \tau\left(T_{0}, \eta^{\prime}\right)\left[\right.\right.$. In particular $\left(x_{S}^{\prime}, v_{S}^{\prime}\right)$ is $\mathcal{F}_{S}^{\prime}$-measurable. We are going to estimate $\tau\left(T_{0}, \eta^{\prime}\right)$ from below: on $\left\{\tau\left(T_{0}, \eta^{\prime}\right)<T\right\}$, $x^{\prime}\left(\tau\left(T_{0}, \eta^{\prime}\right)\right)$ is almost surely at least at a distance $r-r^{\prime}$ from $x^{\prime}\left(T_{0}\right)$. By the triangle inequality,

$$
\int_{T_{0}}^{\tau\left(T_{0}, \eta^{\prime}\right)}\left\|v^{\prime}(s)\right\|_{x^{\prime}(s)} \mathrm{d} s \geqslant r-r^{\prime} .
$$

Denoting by $\tau_{1}=\tau\left(T_{0}, \eta^{\prime}\right)$, we obtained thus a solution $\left(x_{t}^{\prime}, v_{t}^{\prime}\right)_{T_{0} \leqslant t<\tau_{1}}$ of (2.1) with initial condition $\eta^{\prime} \in T M$ at time $T_{0}$. We can apply the previous process with $\eta^{\prime}=v^{\prime}\left(\tau_{1}\right)$ and $T_{0}=\tau_{1}$ and define then a $\mathcal{F}_{t}^{\prime}$-stopping time $\tau_{2}$ and so on. We construct thus a non-decreasing sequence $\left(\tau_{n}\right)_{n}$ of stopping time, which tends to a 
stopping time $\tau$. By similar calculations to those presented in lemma 5.1, we can show that, for $t<T$ and for all $n$,

$$
\mathbb{E} \sup _{0 \leqslant s \leqslant t}\left\|v_{s \wedge \tau_{n}}^{\prime}\right\|_{x^{\prime}(s)}^{2} \leqslant C
$$

Moreover, by (6.2) applied on each $\left[\tau_{i}, \tau_{i+1}\left[\right.\right.$, we have for all stopping time $S \in\left[T_{0}, T[\right.$,

$$
C \geqslant \mathbb{E} \int_{T_{0}}^{S}\left\|v^{\prime}(s)\right\|_{x^{\prime}(s)} \mathrm{d} s \geqslant \int_{\left\{\tau_{n} \leqslant S\right\}} n\left(r-r^{\prime}\right) \mathrm{d} \mathbb{P},
$$

and then

$$
\lim _{n \rightarrow+\infty} \mathbb{P}\left(\left\{\tau_{n} \leqslant S\right\}\right)=0
$$

which proves that $\tau=T$.

\section{Application to the pendulum}

We consider a spherical pendulum (see figure 1) consisting of a material point with unit mass attached to a rigid stem of length 1 and of negligible mass, which is connected at its other end to a fixed point $O$ by a connection authorizing only angular displacements. In our units, the gravity is 1 . In addition to its weight, the pendulum is submitted, at its mobile end, to a dry friction of Coulomb type (with coefficient $\mu$ ) and a random force acting in the tangent plane of the sphere. We will now define precisely our model of stochastic and dry friction forces. The initial conditions are $\left(x_{0}, v_{0}\right) \in T \mathbb{S}^{2}$.

In the present case, the Riemannian metric is the metric induced by the Euclidean metric of $\mathbb{R}^{3}$ and, therefore, we will abuse notation and denote the scalar product in the tangent space at $x$ to $\mathbb{S}^{2}$ without an index $x$, the same convention holding for the norm of tangent vectors.

Let $H_{1}$ and $H_{2}$ be two sections of $T \mathbb{S}^{2}$ which satisfy the following conditions.

\section{Assumption 7.1.}

$$
\begin{aligned}
& \forall x \in \mathbb{S}^{2},\left\|H_{1}(x)\right\|_{x}+\left\|H_{2}(x)\right\|_{x} \leqslant C, \\
& \forall x, y \in \mathbb{S}^{2} \text { in the same open hemisphere } \\
& \left\|H^{1}(x)-\bar{\tau}(y, x) H^{1}(y)\right\|_{x}+\left\|H^{2}(x)-\bar{\tau}(y, x) H^{2}(y)\right\|_{x} \leqslant C d_{\mathbb{S}^{2}}(x, y) .
\end{aligned}
$$

Observe that (7.2) implies the continuity of $H_{1}$ and $H_{2}$; therefore, according to the theorem of the hairy sphere, each of the fields $H_{1}$ and $H_{2}$ has to vanish at some point of $\mathbb{S}^{2}$.

Let $\left(W^{1}, W^{2}\right)$ be a two-dimensional Brownian motion; we formally write the stochastic part of the exterior forces as

$$
F_{s}(t, x) \mathrm{d} t=\mathrm{d} W^{1}(t) H_{1}(x)+\mathrm{d} W^{2}(t) H_{2}(x) .
$$

Thanks to assumptions (7.1) and (7.2), the linear mapping

$$
\sigma^{\prime}(x)\left(v^{1}, v^{2}\right)=v^{1} H_{1}(x)+v^{2} H_{2}(x)
$$

satisfies assumptions 2.3 and 2.4 of $\S 2$. 
If $t \mapsto x(t)$ is a motion on the sphere $\mathbb{S}^{2}$, we differentiate twice the relation $\|x(t)\|^{2}=1$ and we find $x \cdot \ddot{x}=-|\dot{x}|^{2}$, which means that the normal component of the acceleration is $a_{\mathrm{N}}(x, v)=-\|v\|^{2} x$.

The normal component of the weight at $x$ is $P_{\mathrm{N}}(x)=p^{n}(x) x$, with $p^{n}(x)$ equal to $x_{3}$. Therefore, the sum of the reaction of the support and of the tension of the stem is

$$
R(x, v)+F(x, v)=x\left(x_{3}+\|v\|^{2}\right) .
$$

The friction force is then given by the multivalued relation

$$
F_{\mathrm{r}}(x, v) \in \begin{cases}\{0\} & \text { if } p^{n}(x) \leqslant-\|v\|^{2}, \\ -\mu\left\{\left(p^{n}(x)+\|v\|^{2}\right) v /\|v\|\right\} & \text { if } p_{n}(x)>-\|v\|^{2} \text { and } v \neq 0, \\ \mu\left\{u \in T_{x} \mathbb{S}^{2},\|u\| \leqslant p^{n}(x),\right\} & \text { if } p^{n}(x)>0 \text { and } v=0 .\end{cases}
$$

Let us check now that $-F_{\mathrm{r}}$ is a monotone operator in the fibre, i.e. for all $w \in$ $-F_{\mathrm{r}}(x, v)$ and $w^{\prime} \in-F_{\mathrm{r}}\left(x, v^{\prime}\right)$, the following inequality holds:

$$
\left(w-w^{\prime}, v-v^{\prime}\right) \geqslant 0 .
$$

If $v$ and $v^{\prime}$ are both in case 1 or 3 of (7.3), it is plain that (7.4) holds. If $w$ is in case 2 or 3 , and $w^{\prime}$ is in case 1 , the conclusion is still clear; therefore, we just have to check the inequality when $v$ and $v^{\prime}$ are in case 2 , or when $v$ is in case 2 and $v^{\prime}$ is in case 3 .

If neither $v$ nor $v^{\prime}$ vanish and $p_{n}(x)>-\min \left(\|v\|^{2},\left\|v^{\prime}\right\|^{2}\right)$, then

$$
\left(w-w^{\prime}, v-v^{\prime}\right)=p^{n}(x)\left(\left(\frac{v}{\|v\|}-\frac{v^{\prime}}{\left\|v^{\prime}\right\|}\right), v-v^{\prime}\right)+\left(\|v\| v-\left\|v^{\prime}\right\| v^{\prime}, v-v^{\prime}\right) .
$$

An elementary calculation shows that the above expression is non-negative.

If $v$ is in the second case and $v^{\prime}$ in the third, we have to study the expression

$$
\left(\left(p^{n}(x)+\|v\|^{2}\right) \frac{v}{\|v\|}-u, v\right)
$$

where $\|u\| \leqslant p^{n}(x)$. It is once again straightforward to verify that this expression is non-negative.

If we put $\nu^{\prime}(x, v)=P_{\mathrm{T}}(x)=-x+x_{3} x$, which is the tangential component of the weight, the equation of the dynamic applied to the pendulum takes the form:

$$
\begin{aligned}
& \mathrm{d} x_{t}=v_{t} \mathrm{~d} t, \\
& D_{\dot{x}(t)} v_{t}+A^{\prime}\left(x_{t}, v_{t}\right) \mathrm{d} t \ni \nu^{\prime}\left(x_{t}, v_{t}\right) \mathrm{d} t+\sigma^{\prime}\left(x_{t}\right) \mathrm{d} W_{t}, \quad 0 \leqslant t<T, \\
& (x(0), v(0))=\left(x_{0}, v_{0}\right) .
\end{aligned}
$$

Remark 7.2. Observe that we could have considered more general forces than the weight, which can be easily included in this theory.

Thanks to remark 2.8 and, since assumptions $2.3-2.5$ are clearly verified, we then have the existence and uniqueness of solutions to equation (7.5).

\section{Conclusion}

In this work a new result on stochastic differential equations is given: the existence and uniqueness of solutions to MSDEs on Riemannian manifolds are obtained. This 
work generalizes the results of Cépa (1998) devoted to the Euclidean case. To simplify the expression of Stratonovich's covariant differential in the equation relative to velocities, that is, to eliminate the quadratic terms, which offers the possibility to approximate equations by classical (with Lipschitz continuous assumptions) MSDE on Euclidean space, we used the parallel transport along the solution curve, but we had to pay by losing Lipschitz continuity of $\tilde{b}$. Then we had to approximate $\tilde{b}$ by Lipschitz continuous maps $b_{n}$ and this allowed us to construct the expected solution. From the application viewpoint the result obtained in this article finds its interest in the modelling of mechanical systems submitted to stochastic forcing and friction; the example of the stochastic spherical pendulum with friction fits into this framework.

To approximate the solution it is possible to adapt a numerical scheme developed for classical stochastic differential equations with convex constraints in Pettersson (2000) and for general MSDE in Bernardin (2003). Numerical simulation of this class of models is the topic of future work.

The authors express their thanks to Professor J. Picard and Professor A. Lachal for their suggestions and encouragement.

\section{References}

Abraham, R. \& Marsden, J. E. 1985 Foundations of mechanics. Addison-Wesley.

Ballard, P. 2002 Formulation and well-posedness of the dynamics of rigid bodies with unilateral or frictional constraints. In Advances in mechanics and mathematics, pp. 3-88. Dordrecht: Kluwer Academic.

Bernardin, F. 2003 Multivaled stochastic differential equations: convergence of a numerical scheme. Set-Valued Analysis 11, 393-415.

Brézis, H. 1973 Opérateurs maximaux monotones et semi-groupes de contractions dans les espaces de Hilbert. Amsterdam: North-Holland.

Cépa, E. 1995 Equations différentielles stochastiques multivoques. Lecture Notes in Mathematics, Séminaire de Probabilités, vol. XXIX, pp. 86-107. Springer.

Cépa, E. 1998 Problème de Skorohod multivoque. Ann. Probab. 26, 500-532.

Emery, M. 1989 Stochastic calculus in manifolds. Springer.

Ikeda, N. \& Watanabe, S. 1989 Stochastic differential equations and diffusion processes, 2nd edn. Amsterdam: North-Holland.

Karatzas, I. \& Shreve, E. 1991 Brownian motion and stochastic calculus, 2nd edn. Springer.

Mao, X. 1997 Stochastic differential equations and applications. Horwood.

Norris, J. R. 1992 A complete differential formalism for stochastic calculus in manifolds. Lecture Notes in Mathematics, Séminaire de Probabilités, vol. XXVI, pp. 189-209. Springer.

Pettersson, R. 2000 Projection scheme for stochastic differential equations with convex constraints. Stoch. Process. Applic. 88, 125-134.

Saisho, Y. 1987 Stochastic differential equations for multi-dimensional domain with reflecting boundary. Probab. Theory Relat. Fields 74, 455-477. 